\title{
MSSM Higgs boson searches at the LHC: benchmark scenarios after the discovery of a Higgs-like particle
}

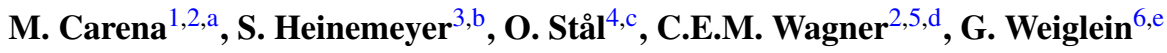 \\ ${ }^{1}$ Theoretical Physics Department, Fermilab, Batavia, IL 60510-0500, USA \\ ${ }^{2}$ Enrico Fermi Institute and Kavli Institute for Cosmological Physics, Department of Physics, the University of Chicago, 5640 Ellis Ave., \\ Chicago, IL 60637, USA \\ ${ }^{3}$ Instituto de Física de Cantabria (CSIC-UC), 39005 Santander, Spain \\ ${ }^{4}$ The Oskar Klein Centre, Department of Physics, Stockholm University, 10691 Stockholm, Sweden \\ ${ }^{5}$ HEP Division, Argonne Natl. Lab., 9700 Cass Ave., Argonne, IL 60439, USA \\ ${ }^{6}$ DESY, Notkestraße 85, 22607 Hamburg, Germany
}

Received: 27 March 2013 / Revised: 12 July 2013 / Published online: 18 September 2013

(c) Springer-Verlag Berlin Heidelberg and Società Italiana di Fisica 2013

\begin{abstract}
A Higgs-like particle with a mass of about 125.5 $\mathrm{GeV}$ has been discovered at the LHC. Within the current experimental uncertainties, this new state is compatible with both the predictions for the Standard Model (SM) Higgs boson and with the Higgs sector in the Minimal Supersymmetric Standard Model (MSSM). We propose new low-energy MSSM benchmark scenarios that, over a wide parameter range, are compatible with the mass and production rates of the observed signal. These scenarios also exhibit interesting phenomenology for the MSSM Higgs sector. We propose a slightly updated version of the well-known $m_{h}^{\max }$ scenario, and a modified scenario $\left(m_{h}^{\bmod }\right)$, where the light $\mathcal{C P}$-even Higgs boson can be interpreted as the LHC signal in large parts of the $M_{A}-\tan \beta$ plane. Furthermore, we define a light stop scenario that leads to a suppression of the lightest $\mathcal{C P}$-even Higgs gluon fusion rate, and a light stau scenario with an enhanced decay rate of $h \rightarrow \gamma \gamma$ at large $\tan \beta$. We also suggest a $\tau$-phobic Higgs scenario in which the lightest Higgs can have suppressed couplings to down-type fermions. We propose to supplement the specified value of the $\mu$ parameter in some of these scenarios with additional values of both signs. This has a significant impact on the interpretation of searches for the non-SM-like MSSM Higgs bosons. We also discuss the sensitivity of the searches to heavy Higgs decays into light charginos and neutralinos, and to decays of the form
\end{abstract}

\footnotetext{
a e-mail: carena@fnal.gov

b e-mail: Sven.Heinemeyer@cern.ch

ce-mail: oscar.stal@fysik.su.se

de-mail: cwagner@hep.anl.gov

e e-mail: Georg.Weiglein@ desy.de
}

$H \rightarrow h h$. Finally, in addition to all the other scenarios where the lightest $\mathcal{C P}$-even Higgs is interpreted as the LHC signal, we propose a low- $M_{H}$ scenario, where instead the heavy $\mathcal{C P}$-even Higgs boson corresponds to the new state around $125.5 \mathrm{GeV}$.

\section{Introduction}

Elucidating the mechanism that controls electroweak symmetry breaking (EWSB) is one of the main tasks of the LHC. The spectacular discovery of a Higgs-like particle with a mass around $125-126 \mathrm{GeV}$, announced by the ATLAS and CMS experiments $[1,2]$, marks a milestone of an effort that has been ongoing for almost half a century and opens a new era of particle physics. Both experiments reported a clear excess in the two photon channel as well as in the $Z Z^{(*)}$ channel, whereas the analyses in other channels have a lower mass resolution and are, at present, less significant. The measured mass varies somewhat between the different channels, and between the two experiments. We shall use the average value $M_{H}^{\text {obs }}=125.5 \pm 1 \mathrm{GeV}$ in the following discussion. The combined sensitivity in each of the experiments reaches more than $5 \sigma$. The central value for the observed rate in the $\gamma \gamma$ channel is above the expectation for a SM Higgs boson in ATLAS results [3], whereas CMS measures a lower rate [4]. Although the statistical significance of possible deviations from the SM prediction is not yet sufficient to draw any definite conclusion, a confirmed deviation in the $\gamma \gamma$ channel with more data could be the first indication of a non-SM nature of the new state, and of possible new physics at the weak scale. 
Among the most studied candidate theories for EWSB in the literature are the Higgs mechanism within the Standard Model (SM) [5-7] and the Minimal Supersymmetric Standard Model (MSSM) [8-10]. Contrary to the SM, two Higgs doublets are required in the MSSM, resulting in five physical Higgs boson degrees of freedom. At lowest order, where the MSSM Higgs sector is $\mathcal{C P}$-conserving, the five physical states are the light and heavy $\mathcal{C} \mathcal{P}$-even Higgs bosons, $h$ and $H$, the $\mathcal{C P}$-odd Higgs boson, $A$, and the charged Higgs boson pair, $H^{ \pm}$. The Higgs sector of the MSSM can be specified at lowest order in terms of the $Z$ boson mass, $M_{Z}$, the $\mathcal{C} \mathcal{P}$-odd Higgs mass, $M_{A}$ (or the charged Higgs mass, $M_{H^{ \pm}}$), and $\tan \beta \equiv v_{2} / v_{1}$, the ratio of the two Higgs vacuum expectation values. The masses of the $\mathcal{C P}$-even neutral Higgs bosons and the charged Higgs boson can be calculated, including higher-order corrections, in terms of the other MSSM parameters [11-13]. An upper bound for the mass of the lightest MSSM Higgs boson of $M_{h} \lesssim 135 \mathrm{GeV}$ was obtained [14], and the remaining theoretical uncertainty in the calculation of $M_{h}$, from unknown higher-order corrections, was estimated to be up to $3 \mathrm{GeV}$, depending on the parameter region.

Given that the experimental uncertainties on the measurements of the production cross sections times branching ratios are still rather large, sizable deviations of various couplings from the SM values are still possible, and even a Higgs sector that differs very significantly from the SM case can fit the data. In particular, while within the MSSM an obvious possibility is to interpret the new state at about 125.5 $\mathrm{GeV}$ as the light $\mathcal{C} \mathcal{P}$-even Higgs boson [15-34], it was pointed out that at least in principle also a much more exotic interpretation could be possible (within the uncertainties), namely in terms of the heavy $\mathcal{C} \mathcal{P}$-even Higgs boson of the MSSM [15, 20, 21, 35, 36]. In such a case all five Higgs bosons of the MSSM Higgs sector would be light, where the heavy $\mathcal{C P}$-even Higgs boson would have a mass around $125.5 \mathrm{GeV}$ and behave roughly $\mathrm{SM}$-like, while the light $\mathcal{C} \mathcal{P}$ even Higgs boson of the MSSM would have heavily suppressed couplings to gauge bosons and a mass that would be typically below the LEP limit for a SM-like Higgs [37].

In parallel with the exciting discovery, the search for non-standard MSSM Higgs bosons at the LHC has continued. The search for the remaining Higgs bosons is pursued mainly via the channels $(\phi=h, H, A)$ :

$$
\begin{aligned}
& p p \rightarrow \phi \rightarrow \tau^{+} \tau^{-} \quad \text { (inclusive), } \\
& b \bar{b} \phi, \phi \rightarrow \tau^{+} \tau^{-} \quad \text { (with } b \text {-tag), } \\
& b \bar{b} \phi, \phi \rightarrow b \bar{b} \quad(\text { with } b \text {-tag), } \\
& p p \rightarrow t \bar{t} \rightarrow H^{ \pm} W^{\mp} b \bar{b}, H^{ \pm} \rightarrow \tau \nu_{\tau}, \\
& g b \rightarrow H^{-} t \quad \text { or } \quad g \bar{b} \rightarrow H^{+} \bar{t}, H^{ \pm} \rightarrow \tau v_{\tau} .
\end{aligned}
$$

The non-observation of any additional state in these production and decay modes puts by now stringent constraints on the MSSM parameter space, in particular on the values of the tree-level parameters $M_{A}$ (or $M_{H^{ \pm}}$) and $\tan \beta$. Similarly, the non-observation of supersymmetric (SUSY) particles puts relevant constraints on the masses of the first and second generation scalar quarks and the gluino, and to lesser degree on the stop and sbottom masses (see Ref. [38, 39] for a recent summary).

Due to the large number of free parameters, a complete scan of the MSSM parameter space is impractical in experimental analyses and phenomenological studies. Therefore the Higgs search results at LEP were interpreted [40] in several benchmark scenarios [41, 42]. In these scenarios only the two parameters that enter the Higgs sector treelevel predictions, $M_{A}$ and $\tan \beta$, are varied (and the results are usually displayed in the $M_{A}-\tan \beta$ plane), whereas the other SUSY parameters, entering via radiative corrections, are fixed to particular benchmark values which are chosen to exhibit certain features of the MSSM Higgs phenomenology. In particular, in the $m_{h}^{\max }$ scenario the benchmark values have been chosen such that the mass of the light $\mathcal{C P}$-even Higgs boson is maximized for fixed $\tan \beta$ and large $M_{A}$ (the scale of the soft SUSY-breaking masses in the stop and sbottom sectors, which sets the mass scale for the corresponding supersymmetric particles, has been fixed to $1 \mathrm{TeV}$ in this scenario). This scenario is useful to obtain conservative bounds on $\tan \beta$ for fixed values of the top-quark mass [43]. Besides the $m_{h}^{\max }$ scenario and the nomixing scenario, where a vanishing mixing in the stop sector is assumed, the small $\alpha_{\text {eff }}$ scenario and a gluophobic Higgs scenario were investigated [40]. While the latter exhibits a strong suppression of the $g g h$ coupling over large parts of the $M_{A}-\tan \beta$ parameter space, the small $\alpha_{\text {eff }}$ scenario has strongly reduced couplings of the light $\mathcal{C P}$-even Higgs boson to down-type fermions for $M_{A} \lesssim 350 \mathrm{GeV}$. This set of benchmark scenarios [41, 42], which was originally proposed in view of the phenomenology of the light $\mathcal{C P}$-even Higgs boson, was subsequently used also for analyses at the Tevatron and at the LHC in the search for the heavier MSSM Higgs bosons. Once the radiative corrections to the bottom mass, commonly denoted by $\Delta_{b}$, are included (see below) the predictions for the channels used for the heavy Higgs searches are affected by a relevant dependence on the higgsino mass parameter $\mu$. Hence, it was proposed to augment the original benchmark values of the $m_{h}^{\max }$ and no-mixing scenarios with a variation of $\mu$ over several discrete values (involving both signs of $\mu$ ) [44].

The existing benchmark scenarios have provided a useful framework for presenting limits from MSSM Higgs searches at LEP, the Tevatron and the LHC, but those benchmark scenarios do not necessarily permit an interpretation of the observed signal of a Higgs-like state at $\sim 125.5 \mathrm{GeV}$ as one of the (neutral) Higgs bosons of the MSSM Higgs sector. In particular, the $m_{h}^{\max }$ scenario has been designed 
such that the higher-order corrections maximize the value of $M_{h}$. As a consequence, over large parts of its parameter space this scenario yields values of the light $\mathcal{C} \mathcal{P}$-even Higgs boson mass above the observed mass of the signal of about $125.5 \mathrm{GeV}$. On the other hand, the no-mixing scenario yields $M_{h} \lesssim 122 \mathrm{GeV}$, so that this scenario does not permit the interpretation of the observed signal in terms of the light $\mathcal{C P}$-even Higgs boson of the MSSM. Also the other two scenarios, small $\alpha_{\text {eff }}$ and the gluophobic Higgs, turn out to be incompatible with $M_{h} \sim 125.5 \mathrm{GeV}$.

In the present paper we therefore propose an update of the MSSM Higgs benchmark scenarios in which we adapt them to the present experimental knowledge and ongoing searches. The scenarios that we are going to propose are defined such that over large parts of their available parameter space the observed signal at about $125.5 \mathrm{GeV}$ can be interpreted in terms of one of the (neutral) Higgs bosons, while the scenarios exhibit interesting phenomenology for the MSSM Higgs sector.

The benchmark scenarios are all specified using lowenergy MSSM parameters; we do not assume any particular soft supersymmetry-breaking scenario. We take into account in detail the constraints from direct searches for Higgs bosons, and we select parameters which lead to consistency with the current bounds on direct searches for supersymmetric particles. Indirect constraints from requiring the correct cold dark matter density, $\mathrm{BR}(b \rightarrow s \gamma), \mathrm{BR}\left(B_{s} \rightarrow \mu^{+} \mu^{-}\right)$ or $(g-2)_{\mu}$, however interesting, depend to a large extent on other parameters of the theory that are not crucial for Higgs phenomenology. Following the spirit of the previous benchmark proposals of Refs. [41, 42, 44] we therefore do not impose any additional constraints of this kind. The scenarios below are defined for the MSSM with real parameters. While an extension to complex parameters and their respective impact on the phenomenology is interesting, it is beyond the scope of the present paper.
The paper is organized as follows: Section 2 gives a summary of the properties of the MSSM Higgs sector and their dependence on the supersymmetric parameters. In particular, we review briefly the most important radiative corrections to the relevant Higgs boson production cross sections and decay widths. In Sect. 3 we propose new MSSM benchmark scenarios, which update and extend the previous benchmark proposals. We discuss the most relevant features of current constraints from the LHC searches for SM-like and non-standard Higgs bosons for each benchmark scenario, including the discovery of a Higgs-like particle with a mass around $125.5 \mathrm{GeV}$. The conclusions are presented in Sect. 4.

\section{Theoretical basis}

\subsection{Notation}

In the description of our notation we are including the complex phases of the relevant SUSY parameters. However, as indicated above, for the definition of the benchmark scenarios we restrict ourselves to the $\mathcal{C P}$-conserving MSSM, i.e. to the case of real parameters. The tree-level masses of the $\mathcal{C P}$-even MSSM Higgs bosons, $M_{h}^{\text {tree }}$ and $M_{H}^{\text {tree }}$, are determined by $\tan \beta$, the $\mathcal{C P}$-odd Higgs boson mass, $M_{A}$, and the $Z$ boson mass, $M_{Z}$. The mass of the charged Higgs boson, $M_{H^{ \pm}}^{\text {tree }}$, is determined from $M_{A}$ and the $W$ boson mass, $M_{W}$, by the relation $\left(M_{H^{ \pm}}^{\text {tree }}\right)^{2}=M_{A}^{2}+M_{W}^{2}$. The main radiative correction to the Higgs boson masses arise from the $t / \tilde{t}$ sector, and for large values of $\tan \beta$ also from the $b / \tilde{b}$ and $\tau / \tilde{\tau}$ sectors, see Refs. [11-13] for reviews.

The mass matrices for the stop and sbottom sectors of the MSSM, in the basis of the current eigenstates $\tilde{t}_{L}, \tilde{t}_{R}$ and $\tilde{b}_{L}, \tilde{b}_{R}$, are given by

$$
\begin{aligned}
& \mathcal{M}_{\tilde{t}}^{2}=\left(\begin{array}{cc}
M_{\tilde{t}_{L}}^{2}+m_{t}^{2}+\cos 2 \beta\left(\frac{1}{2}-\frac{2}{3} s_{\mathrm{w}}^{2}\right) M_{Z}^{2} & m_{t} X_{t}^{*} \\
m_{t} X_{t} & M_{\tilde{t}_{R}}^{2}+m_{t}^{2}+\frac{2}{3} \cos 2 \beta s_{\mathrm{w}}^{2} M_{Z}^{2}
\end{array}\right), \\
& \mathcal{M}_{\tilde{b}}^{2}=\left(\begin{array}{cc}
M_{\tilde{b}_{L}}^{2}+m_{b}^{2}+\cos 2 \beta\left(-\frac{1}{2}+\frac{1}{3} s_{\mathrm{w}}^{2}\right) M_{Z}^{2} & m_{b} X_{b}^{*} \\
m_{b} X_{b} & M_{\tilde{b}_{R}}^{2}+m_{b}^{2}-\frac{1}{3} \cos 2 \beta s_{\mathrm{w}}^{2} M_{Z}^{2}
\end{array}\right),
\end{aligned}
$$

where

$$
\begin{aligned}
& m_{t} X_{t}=m_{t}\left(A_{t}-\mu^{*} \cot \beta\right), \\
& m_{b} X_{b}=m_{b}\left(A_{b}-\mu^{*} \tan \beta\right) .
\end{aligned}
$$

Here $A_{t}$ denotes the trilinear Higgs-stop coupling, $A_{b}$ denotes the Higgs-sbottom coupling, and $\mu$ is the higgsino mass parameter. We furthermore use the notation $s_{\mathrm{W}}=$ $\sqrt{1-c_{\mathrm{w}}^{2}}$, with $c_{\mathrm{w}}=M_{W} / M_{Z}$.

$\mathrm{SU}(2)$ gauge invariance leads to the relation

$M_{\tilde{t}_{L}}=M_{\tilde{b}_{L}}$.

We shall concentrate on the case

$M_{\tilde{t}_{L}}=M_{\tilde{b}_{L}}=M_{\tilde{t}_{R}}=M_{\tilde{b}_{R}}=: M_{\mathrm{SUSY}}$. 
This identification of the diagonal elements of the third generation squark mass matrices leads to a simple phenomenological characterization of the third generation squark effects. The relaxation of this condition to the case where $M_{\tilde{t}_{R}} \neq M_{\tilde{t}_{L}} \neq M_{\tilde{b}_{R}}$, has been studied, for instance, in Ref. [45-47]. In the case of Eq. (9), the most important parameters for the corrections in the Higgs sector are $m_{t}$, $M_{\text {SUSY }}, X_{t}$, and $X_{b}$.

Similarly, the corresponding soft SUSY-breaking parameters in the scalar tau/neutrino sector are denoted as $A_{\tau}$ and $M_{\tilde{l}_{3}}$, where we assume the diagonal soft SUSY-breaking entries in the stau/sneutrino mass matrices to be equal to each other as we $\operatorname{did}$ in the $\tilde{t} / \tilde{b}$ sector. For the squarks and sleptons of the first and second generations we also assume equality of the diagonal soft SUSY-breaking parameters, denoted as $M_{\tilde{q}_{1,2}}$ and $M_{\tilde{l}_{1,2}}$, respectively. The off-diagonal $A$-terms always appear multiplied with the corresponding fermion mass. Hence, for the definition of the benchmark scenarios the $A$-terms associated with the first and second sfermion generations have a negligible impact and can be set to zero for simplicity.

The Higgs sector depends also on the gaugino masses. For instance, at the two-loop level the gluino mass, $m_{\tilde{g}}$, enters the predictions for the Higgs boson masses. The Higgs sector observables furthermore depend on the $\mathrm{SU}(2)$ and $\mathrm{U}(1)$ gaugino mass parameters, $M_{2}$ and $M_{1}$, respectively, which are usually assumed to be related via the GUT relation,

$M_{1}=\frac{5}{3} \frac{s_{\mathrm{w}}^{2}}{c_{\mathrm{w}}^{2}} M_{2}$.

\subsection{Higgs mass calculations and their scheme dependence}

Corrections to the MSSM Higgs boson sector have been evaluated in several approaches, see, e.g. Ref. [48]. The remaining theoretical uncertainty on the light $\mathcal{C P}$-even Higgs boson mass has been estimated to be $\Delta M_{h}^{\text {theory }} \lesssim 3 \mathrm{GeV}$ depending on the parameter region $[13,14]$. The leading and subleading parts of the existing two-loop calculations have been implemented into public codes. The program FeynHiggs [14, 46, 49-51] is based on results obtained in the Feynman-diagrammatic (FD) approach, while the code CPsuperH [52-54] is based on results obtained using the renormalization group ( $R G)$ improved effective potential approach [48, 55-58]. For the MSSM with real parameters the two codes can differ by a few $\mathrm{GeV}$ for the prediction of $M_{h}$, partly due to formally subleading two-loop corrections that are included only in FeynHiggs. Both codes do not incorporate the subleading two-loop contributions evaluated in Ref. [59-66], which are not available in a readily usable code format. The existing three-loop corrections evaluated in Refs. $[67,68]$ are also not included, since they are not available in a format that can be added straight-forwardly to the existing calculations (see, however, Ref. [69]).

It is important to stress that the FD results have been obtained in the on-shell (OS) renormalization scheme, whereas the RG results have been calculated using the $\overline{\mathrm{MS}}$ scheme; a detailed comparison of the results in the two schemes is presented in Refs. [48, 70] (see also Refs. [71, 72]). Therefore, the parameters $X_{t}$ and $M_{\text {SUSY }}$ (which are most important for the corrections in the Higgs sector) are scheme-dependent and thus differ in the two approaches. The differences between the corresponding parameters have to be taken into account when comparing the results. Considering the dominant standard QCD and SUSY-QCD corrections at the oneloop level, the relations between the stop mass parameters in the two different schemes are given by [48]

$$
\begin{aligned}
M_{S}^{2, \overline{\mathrm{MS}}} & \approx M_{S}^{2, \mathrm{OS}}-\frac{8}{3} \frac{\alpha_{s}}{\pi} M_{S}^{2}, \\
X_{t}^{\overline{\mathrm{MS}}} \approx & X_{t}^{\mathrm{OS}}+\frac{\alpha_{s}}{3 \pi} \\
& \times M_{S}\left(8+4 \frac{X_{t}}{M_{S}}-\frac{X_{t}^{2}}{M_{S}^{2}}-3 \frac{X_{t}}{M_{S}} \log \left(\frac{m_{t}^{2}}{M_{S}^{2}}\right)\right),
\end{aligned}
$$

where $M_{S}^{2}:=M_{\mathrm{SUSY}}^{2}+m_{t}^{2}$. In these relations we have assumed $m_{\tilde{g}}=M_{\text {SUSY }}$. It should be noted that it is not necessary to distinguish between $\overline{\mathrm{MS}}$ and on-shell quantities in the terms proportional to $\alpha_{s}$, since this difference is of higher order. The change of scheme induces in general only a minor shift, of the order of $4 \%$, in the parameter $M_{\text {SUSY }}$, but sizable differences can occur between the numerical values of $X_{t}$ in the two schemes, see Refs. [46, 48, 72].

\subsection{Leading effects from the bottom/sbottom sector}

At tree level, the bottom-quark Yukawa coupling, $h_{b}$, controls the interaction between the Higgs fields and the sbottom quarks and determines the bottom-quark mass $m_{b}=$ $h_{b} v_{1}$. This relation is affected at one-loop order by large radiative corrections proportional to $h_{b} v_{2}$ [73-78], thereby giving rise to $\tan \beta$-enhanced contributions. These terms, which are often called threshold corrections to the bottomquark mass or $\Delta_{b}$ corrections, may be generated by gluinosbottom one-loop diagrams (resulting in $\mathcal{O}\left(\alpha_{b} \alpha_{s}\right)$ corrections to the Higgs masses, where $\alpha_{b}=h_{b}^{2} / 4 \pi$ ), by charginostop loops (giving $\mathcal{O}\left(\alpha_{b} \alpha_{t}\right)$ corrections, where $\alpha_{t}=h_{t}^{2} / 4 \pi$ ), or by other subleading contributions. At sufficiently large values of $\tan \beta$, the $\tan \beta$-enhancement may compensate the loop suppression, and these contributions may be numerically relevant. Therefore, an accurate determination of $h_{b}$ from the experimental value of the bottom-quark mass requires a resummation of these threshold effects to all orders in the perturbative expansion [76, 77].

The leading $\Delta_{b}$-induced effects on the Higgs couplings may be included in an effective Lagrangian formalism [76, 
$79,80]$. Numerically this represents the dominant contributions to the Higgs couplings from the sbottom sector (see also [81-84]). The effective Lagrangian is given by

$$
\begin{aligned}
\mathcal{L}= & \frac{g}{2 M_{W}} \frac{\bar{m}_{b}}{1+\Delta_{b}}\left[\tan \beta A i, \bar{b} \gamma_{5} b+\sqrt{2} V_{t b} \tan \beta H^{+} \bar{t}_{L} b_{R}\right. \\
& +\left(\frac{\sin \alpha}{\cos \beta}-\Delta_{b} \frac{\cos \alpha}{\sin \beta}\right) h \bar{b}_{L} b_{R} \\
& \left.-\left(\frac{\cos \alpha}{\cos \beta}+\Delta_{b} \frac{\sin \alpha}{\sin \beta}\right) H \bar{b}_{L} b_{R}\right]+ \text { h.c. }
\end{aligned}
$$

Here $\bar{m}_{b}$ denotes the running bottom-quark mass at the chosen scale including SM QCD corrections. The prefactor $1 /\left(1+\Delta_{b}\right)$ in Eq. (13) arises from the resummation of the leading corrections to all orders. The additional terms proportional to $\Delta_{b}$ in the $h \bar{b} b$ and $H \bar{b} b$ couplings arise from the mixing between the $\mathcal{C} \mathcal{P}$-even Higgs bosons and from the one-loop coupling of the bottom quark to $H_{u}$ (the doublet that gives masses to the up-type fermions).

As stressed above there are two main contributions to the threshold correction $\Delta_{b}$, an $\mathcal{O}\left(\alpha_{s}\right)$ correction from a sbottom-gluino loop and an $\mathcal{O}\left(\alpha_{t}\right)$ correction from a stophiggsino loop. In the limit of $M_{S} \gg m_{t}$ and $\tan \beta \gg 1$, taking these two contributions into account ${ }^{1} \Delta_{b}$ reads [73-75]

$$
\begin{aligned}
\Delta_{b}= & \frac{2 \alpha_{s}}{3 \pi} m_{\tilde{g}} \mu \tan \beta \times I\left(m_{\tilde{b}_{1}}, m_{\tilde{b}_{2}}, m_{\tilde{g}}\right)+\frac{\alpha_{t}}{4 \pi} A_{t} \mu \tan \beta \\
& \times I\left(m_{\tilde{t}_{1}}, m_{\tilde{t}_{2}}, \mu\right) .
\end{aligned}
$$

The function $I$ is given by

$$
\begin{aligned}
I(a, b, c)= & \frac{1}{\left(a^{2}-b^{2}\right)\left(b^{2}-c^{2}\right)\left(a^{2}-c^{2}\right)} \\
& \times\left(a^{2} b^{2} \log \frac{a^{2}}{b^{2}}+b^{2} c^{2} \log \frac{b^{2}}{c^{2}}+c^{2} a^{2} \log \frac{c^{2}}{a^{2}}\right) \\
& \sim \frac{1}{\max \left(a^{2}, b^{2}, c^{2}\right)} .
\end{aligned}
$$

The $\Delta_{b}$ correction can become very important for large values of $\tan \beta$ and the ratios of $\mu m_{\tilde{g}} / M_{\text {SUSY }}^{2}$ and $\mu A_{t} / M_{\text {SUSY }}^{2}$. While for $\mu, m_{\tilde{g}}, A_{t}>0$, the $\Delta_{b}$ correction is positive, leading to a suppression of the bottom Yukawa coupling, for negative values of $\Delta_{b}$ the bottom Yukawa coupling may be strongly enhanced and can even acquire nonperturbative values when $\Delta_{b} \rightarrow-1$.

The impact of the $\Delta_{b}$ corrections on the searches for the heavy MSSM Higgs bosons has been analyzed in Ref. [44]

\footnotetext{
${ }^{1}$ The evaluation in FeynHiggs that we shall use in our numerical computations contains the full one-loop contributions to $\Delta_{b}$ as given in Ref. [85]. The leading QCD two-loop corrections to $\Delta_{b}$ are also available [86, 87]; they stabilize the scale dependence of $\Delta_{b}$ substantially. Corrections in the MSSM with non-minimal flavor structure were recently published in Ref. [88].
}

(see also Refs. $[89,90])$. It was shown that the exclusion bounds in the channels defined by Eqs. (2) and (3) depend strongly on the sign and size of $\Delta_{b}$, whereas the channels equations (1) and (4) show a weaker dependence on $\Delta_{b}$, as a consequence of a partial cancelation of the $\Delta_{b}$ contributions. In order to demonstrate the phenomenological consequences of varying the parameter $\mu$, it was recommended in Ref. [44] to augment the original benchmark values of the $m_{h}^{\max }$ and no-mixing scenarios [42] with a variation of $\mu$ over discrete values in the range $-1000 \mathrm{GeV}$ to $+1000 \mathrm{GeV}$. When investigating negative values of $\mu$, in particular $\mu=-1000 \mathrm{GeV}$, the considered range of $\tan \beta$ needs to be restricted to sufficiently low values in order to maintain a perturbative behavior of the bottom Yukawa coupling.

\section{Benchmark scenarios}

In the following subsections we propose updated benchmark scenarios, in which the observed LHC signal at $\sim 125.5 \mathrm{GeV}$ can be interpreted as one of the (neutral $\mathcal{C P}$-even) states of the MSSM Higgs sector, and we discuss relevant features of their phenomenology. In particular, within present experimental uncertainties, these benchmark scenarios allow for different interpretations of the production and decay rates of the discovered Higgs-like state. In addition, the scenarios are useful in the search of the other, non-SM-like, MSSM Higgs bosons. For convenience, we also give a table containing the parameter values for all the proposed scenarios in the Appendix.

Concerning the parameters that have only a minor impact on the MSSM Higgs sector predictions, we propose fixing them to the following values:

$M_{\tilde{q}_{1,2}}=1500 \mathrm{GeV}$,

$M_{\tilde{l}_{1,2}}=500 \mathrm{GeV}$,

$A_{f}=0 \quad(f=c, s, u, d, \mu, e)$.

$M_{1}$ is fixed via the GUT relation, Eq. (10). Motivated by the analysis in Ref. [44] we suggest to investigate for each scenario given in Sects. 3.1-3.3, in addition to the default values given there, the following values of $\mu$ :

$\mu= \pm 200, \pm 500, \pm 1000 \mathrm{GeV}$.

These values of $\mu$ allow for both an enhancement and a suppression of the bottom Yukawa coupling, and are consistent with the limits from direct searches for charginos and neutralinos at LEP [91]. As mentioned above, when investigating negative values of $\mu$ the considered range of $\tan \beta$ needs to be restricted to sufficiently low values in order to maintain a perturbative behavior of the bottom Yukawa coupling. 
The value for the top-quark mass used in the original benchmark scenarios $[42,44]$ was chosen according to the experimental central value at that time. For the new scenarios we propose to substitute this value with the most up-todate experimental central value $m_{t}=173.2 \mathrm{GeV}$ [92].

To analyze the benchmark scenarios discussed below, and to generate the MSSM Higgs predictions for the plots, we use FeynHiggs 2.9.4 [14, 46, 49-51]. Here relevant, values for the input parameters are quoted both in the onshell scheme (suitable for FeynHiggs), as well as in the $\overline{\mathrm{MS}}$ scheme. The latter set of parameters can readily be used by CPsuperH [52-54]. Using this code we have verified that these parameter settings lead to similar Higgs phenomenology. ${ }^{2}$ We also show the exclusion bounds (at $95 \%$ C.L.) from direct Higgs searches, evaluated with Higgs Bounds 4.0.0 [94-97] (linked to FeynHiggs). This code uses exclusion limits from LEP, the Tevatron, and the LHC (results presented up until the Moriond 2013 conference are included). In particular this includes the most sensitive limits from searches for neutral $[98,99]$ and charged $[100,101]$ MSSM Higgs bosons, and the combined limits on Higgs bosons with SM-like couplings [1, 102]. For a full list of included limits and references, we refer to Appendix A of Ref. [97]. A combined uncertainty on the SMlike Higgs mass of $\Delta M_{h}=3 \mathrm{GeV}\left(\Delta M_{H}=3 \mathrm{GeV}\right.$ in the last scenario) was used when evaluating the limits. While an estimate of the currently excluded region is given in this way, ${ }^{3}$ we would like to emphasize that a main point of this work is to encourage ATLAS and CMS to perform dedicated searches for MSSM Higgs bosons in these scenarios.

For each benchmark scenario we show the region of parameter space where the mass of the (neutral $\mathcal{C P}$-even) MSSM Higgs boson that is interpreted as the newly discovered state is within the range $125.5 \pm 3 \mathrm{GeV}$ and $125.5 \pm$ $2 \mathrm{GeV}$. The $\pm 3 \mathrm{GeV}$ uncertainty is meant to represent a combination of the present experimental uncertainty of the determined mass value and of the theoretical uncertainty in the MSSM Higgs mass prediction from unknown higherorder corrections. Taking into account a parametric uncertainty from the top-quark mass measurements of $\delta m_{t}^{\exp }=$ $0.9 \mathrm{GeV}$ [92] would result in an even slightly larger interval of "acceptable" $M_{h}$ values, while all other features

\footnotetext{
${ }^{2}$ For calculations of the Higgs branching ratios, there also exist other codes like HDECAY [93]. The branching ratio predictions for the different scenarios are generally in good agreement between the different codes, and we use FeynHiggs for simplicity.

${ }^{3} \mathrm{HiggsBounds}$ provides a compilation of cross section limits obtained from Higgs searches at LEP, the Tevatron and the LHC. For testing whether a particular parameter point of a considered model is excluded, first the search channel with the highest expected sensitivity for an exclusion is determined, and then the observed limit is confronted with the model predictions for this single channel only, see Ref. [94-96] for further details.
}

remain the same. The displayed area with $\pm 3 \mathrm{GeV}$ uncertainty should therefore be viewed as being in (conservative) agreement with a Higgs mass measurement of $\sim 125.5 \mathrm{GeV}$. In particular, in the case that the lightest $\mathcal{C P}$-even Higgs is interpreted as the newly discovered state, the couplings of the $h$ are close to the corresponding SM values (modulo effects from light SUSY particles, see below). Consequently, those rate measurements from the LHC that agree well with the SM are then naturally in good agreement also with the MSSM predictions. The area corresponding to the $\pm 2 \mathrm{GeV}$ uncertainty indicates how the region that is in agreement with the measured value would shrink as a consequence of reducing the theoretical and experimental uncertainties to a combined value of $2 \mathrm{GeV}$.

\subsection{The $m_{h}^{\max }$ scenario}

The $m_{h}^{\max }$ scenario was originally defined to give conservative exclusion bounds on $\tan \beta$ in the LEP Higgs searches [40, 42, 43]. The value of $X_{t}$ was chosen in order to maximize the lightest $\mathcal{C} \mathcal{P}$-even Higgs mass at large values of $M_{A}$ for a given value of $\tan \beta$ (and all other parameters fixed). Taking into account (besides the latest limits from the Higgs searches at the Tevatron and the LHC) the observation of a new state at $\sim 125.5 \mathrm{GeV}$ and interpreting this signal as the light $\mathcal{C P}$-even Higgs, the $m_{h}^{\max }$ scenario can now be used to derive conservative lower bounds on $M_{A}$, $M_{H^{ \pm}}$and $\tan \beta$ [15].

On the other hand, since the $m_{h}^{\max }$ scenario has been designed such that the higher-order corrections maximize the value of $M_{h}$, in the decoupling region $\left(M_{A} \gg M_{Z}\right)$ and for $\tan \beta \gtrsim 10$ this scenario yields $M_{h}$ values that are significantly higher (above $130 \mathrm{GeV}$ ) than the observed mass of the signal. Compatibility of the predicted values for the mass of the light $\mathcal{C} \mathcal{P}$-even Higgs boson with the mass of the observed signal is therefore achieved only in a relatively small region of the parameter space, in particular for rather low values of $\tan \beta$. However, given that the $m_{h}^{\max }$ scenario is useful to provide conservative lower bounds on the parameters determining the MSSM Higgs sector at tree level $\left(M_{A}\right.$ or $M_{H^{ \pm}}$and $\tan \beta$ ) and has widely been used for analyses in the past, we nevertheless regard it as a useful benchmark scenario also for the future. We therefore include a slightly updated version of the $m_{h}^{\max }$ scenario in our list of proposed benchmarks.

We define the parameters of the (updated) $m_{h}^{\max }$ scenario (with the remaining values as defined in the previous section) as follows:

$$
\begin{gathered}
\frac{m_{h}^{\max }:}{m_{t}=173.2 \mathrm{GeV},} \\
M_{\mathrm{SUSY}}=1000 \mathrm{GeV},
\end{gathered}
$$


$\mu=200 \mathrm{GeV}$,

$M_{2}=200 \mathrm{GeV}$,

$X_{t}^{\mathrm{OS}}=2 M_{\text {SUSY }} \quad$ (FD calculation $)$,

$X_{t}^{\overline{\mathrm{MS}}}=\sqrt{6} M_{\text {SUSY }} \quad$ (RG calculation),

$A_{b}=A_{\tau}=A_{t}$,

$m_{\tilde{g}}=1500 \mathrm{GeV}$,

$M_{\tilde{l}_{3}}=1000 \mathrm{GeV}$.

Besides (as mentioned above) using the current experimental central value for the top-quark mass, the most relevant change in the definition of the $m_{h}^{\max }$ scenario is an increased value of the gluino mass, which has been adopted in view of the limits from the direct searches for SUSY particles at the LHC $[38,39]$. It should be noted that slightly higher values of $M_{h}$ can be reached if one uses lower values of $m_{\tilde{g}}$ as input. Consequently, slightly more conservative exclusion bounds on $\tan \beta, M_{A}$ and $M_{H^{ \pm}}$can be obtained if one uses as input the lowest possible value for $m_{\tilde{g}}$ that is still allowed in this scenario by the most up-to-date exclusion bounds from ATLAS and CMS, but with $m_{\tilde{g}} \geq 800 \mathrm{GeV}$. Similarly, more conservative exclusion bounds can of course also be obtained by increasing the input value for $M_{\mathrm{SUSY}}$, for instance by using $M_{\text {SUSY }}=2000 \mathrm{GeV}$ and $m_{\tilde{g}}=0.8 M_{\text {SUSY }}$ (i.e., the "original" setting of $m_{\tilde{g}}$ as defined in Ref. [42]), see below. We encourage the experimental collaborations to take into consideration in their analyses also those extensions of the $m_{h}^{\max }$ scenario.

In Fig. 1 we show the $M_{A}-\tan \beta$ plane (left) and the $M_{H^{ \pm}}-\tan \beta$ plane (right) in the (updated) $m_{h}^{\max }$ scenario. As explained above, the areas marked as excluded in the plots have been determined using HiggsBounds 4.0.0beta [94-96] (linked to FeynHiggs). The blue areas in the figure indicate regions that are excluded by LEP Higgs searches, and the red areas indicate regions that are excluded by LHC searches for a SM Higgs (lighter red) and for (nonstandard) MSSM Higgs bosons (solid red). The solid red region of LHC exclusion in this plane cuts in from the upper left corner, in the region of large $\tan \beta$. The most sensitive processes here are given by Eq. (1). These processes have an enhanced rate growing with $\tan \beta$. The "cutoff" in the excluded region for $M_{A}>800 \mathrm{GeV}$ (corresponding roughly to values of $\tan \beta$ above 50) is due to the fact that no experimental limits for $M_{A}>800 \mathrm{GeV}$ have yet been published.

Furthermore, Fig. 1 shows regions in lighter red ("thin strips" at $\tan \beta$ values close to the LEP limit and moderate to large values of $M_{A}$ and $M_{H^{ \pm}}$), indicating the exclusion of the light $\mathcal{C P}$-even Higgs boson via SM-Higgs searches at the LHC. In this region the LHC extends the LEP exclusion bounds for a SM-like Higgs to higher Higgs boson masses.

The two green colors in Fig. 1 indicate where $M_{h}=$ $125.5 \pm 2$ (3) GeV. As discussed above, the $\pm 3 \mathrm{GeV}$ region should represent a reasonable combination of the current experimental and theoretical uncertainties. The fact that the LHC exclusion region from the SM Higgs searches does not exactly "touch" the green band is a consequence of taking into account the theoretical uncertainties in the prediction for the Higgs boson mass in determining the excluded regions. The incorporation of the theoretical uncertainties is also responsible for the fact that in Fig. 1 there is no excluded region from the SM Higgs searches at the LHC for $\tan \beta$ values above the green region. It may be useful to regard the green region as that favored by the LHC observation, even though other parameter regions exist that are not

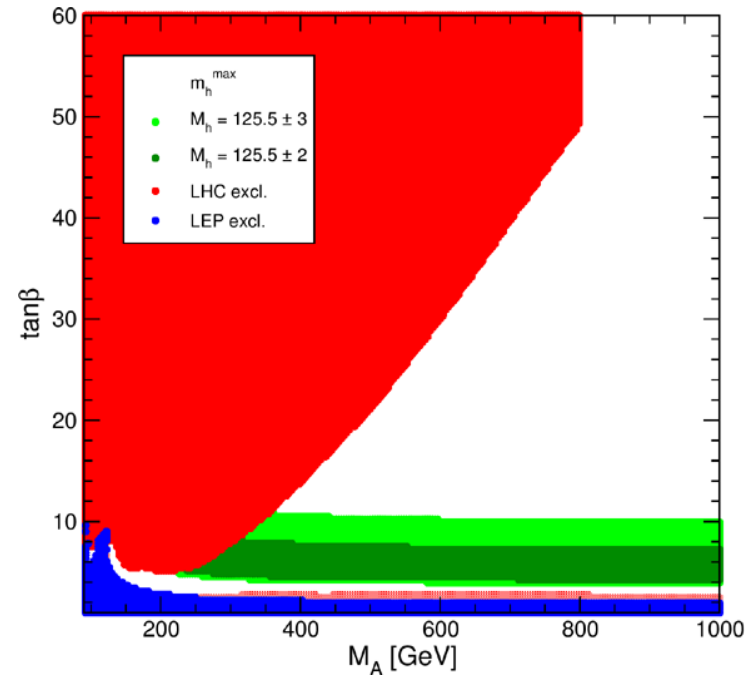

Fig. 1 The $M_{A}-\tan \beta$ (left) and $M_{H^{ \pm}-\tan \beta}$ (right) planes in the (updated) $m_{h}^{\max }$ scenario, with excluded regions from direct Higgs searches at LEP (blue), and the LHC (solid red); the dotted (lighter)

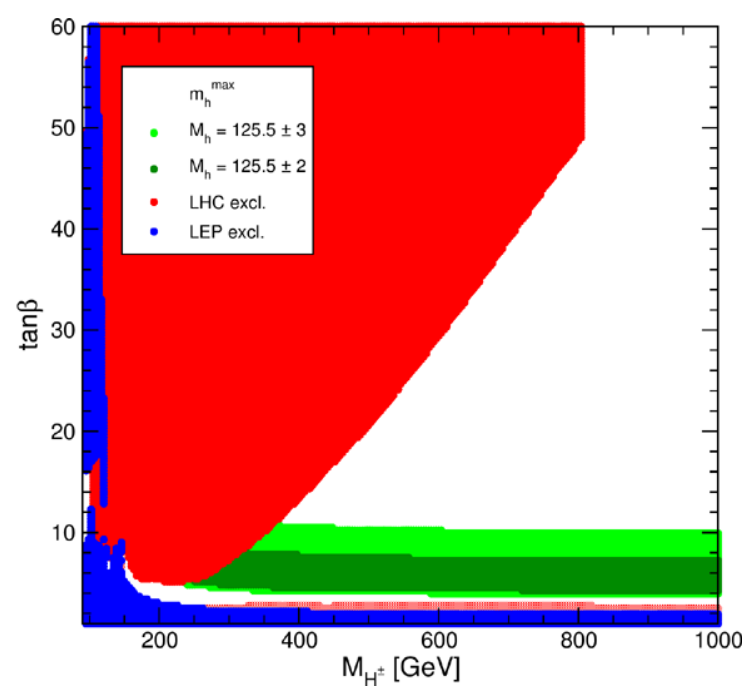

red region is excluded by LHC searches for a SM-like Higgs boson. The two green shades correspond to the parameters for which $M_{h}=125.5 \pm 2(3) \mathrm{GeV}$, see text (Color figure online) 
formally excluded (according to the prescription adopted in HiggsBounds [94-96]). The effects of the theory uncertainty of $\pm 3 \mathrm{GeV}$ used in the evaluation of the experimental bounds are displayed in Fig. 2, where we neglect this theory uncertainty. It can be observed that large parts of the $M_{A}-\tan \beta$ plane (left) and of the $M_{H^{ \pm}}-\tan \beta$ plane (right) would then be excluded in the $m_{h}^{\max }$ scenario from the LHC searches for a SM-like Higgs boson. The resulting excluded region is shown in light red. In particular, for $\tan \beta$ values above the green band the predicted $M_{h}$ value turns out to be too high.

Interpreting the light $\mathcal{C P}$-even Higgs as the new state at $\sim 125.5 \mathrm{GeV}$, a new conservative lower bound on $\tan \beta$ in the MSSM can be obtained from the lowest values on the green bands in Fig. 1 (see Ref. [15] for details). Similarly, the lowest values of $M_{A}$ and $M_{H^{ \pm}}$in the green region (i.e., where the green region touches the excluded region from Higgs searches at the LHC) give a conservative lower bound on these parameters [15]. In particular, from the right plot of Fig. 1 it follows that $M_{H^{ \pm}}<m_{t}$ is excluded for $M_{\text {SUSY }}=1 \mathrm{TeV}$ (if the light $\mathcal{C} \mathcal{P}$-even Higgs is interpreted as the new state at $\sim 125.5 \mathrm{GeV}$ ). Raising $M_{\text {SUSY }}$ to higher values, e.g. to $2000 \mathrm{GeV}$, one finds that $M_{H^{ \pm}}<m_{t}$ might still be marginally allowed. These bounds could be improved by a more precise theoretical prediction and experimental determination of $M_{h}$, and more data on MSSM Higgs boson searches in the region of low values of $M_{A}$ could clearly have an important impact.

It should finally be noted that the sensitivity of the searches for MSSM Higgs bosons in $\tau^{+} \tau^{-}$and $b \bar{b}$ final states that determines the solid red region in Fig. 1 is significantly affected where additional decay modes of the heavy MSSM Higgs bosons are open. In particular, for sufficiently large values of $M_{A}$ decays of the MSSM Higgs bosons $H$ and $A$ into charginos and neutralinos can have an important impact, depending on the parameters in the chargino/neutralino sector. This issue will be discussed in more detail below. Furthermore, interpreting the light $\mathcal{C P}$. even Higgs as the new state at $\sim 125.5 \mathrm{GeV}$ means that the decay $H \rightarrow h h$ is kinematically possible over a large part of the parameter space of the $m_{h}^{\max }$ scenario (and of its variants that will be discussed below). This decay mode can be particularly important in the region of relatively low values of $\tan \beta$ that is favored in the $m_{h}^{\max }$ scenario (see Refs. [72, 103] for details of the calculation). As an example, for $M_{A}=300 \mathrm{GeV}$ and $\tan \beta=7$, i.e. close to the experimental limit from the Higgs searches at the LHC, we find $\operatorname{BR}(H \rightarrow h h)=12 \%$. This branching ratio increases for lower values of $\tan \beta$. For $\tan \beta=4.5$ we find $\mathrm{BR}(H \rightarrow h h)=27 \%$. The two values quoted above are for $M_{2}=200 \mathrm{GeV}$, where also competing decay modes into charginos and neutralinos are open. Increasing the SU(2) gaugino mass parameter to $M_{2}=2000 \mathrm{GeV}$, thus increasing the masses of the charginos and neutralinos, yields $\mathrm{BR}(H \rightarrow h h)=19 \%$ for $\tan \beta=7$ and $\mathrm{BR}(H \rightarrow h h)=$ $50 \%$ for $\tan \beta=4.5$ (for $M_{A}=300 \mathrm{GeV}$, as before). We encourage ATLAS and CMS to enhance the sensitivity of their searches for MSSM Higgs bosons by performing also dedicated searches for Higgs decays into SUSY particles (see the discussion below), where initial analyses can be found, e.g., in Ref. [104-106].

\subsection{The $m_{h}^{\text {mod }}$ scenario}

As explained in the discussion of Fig. 1, the mass of the light $\mathcal{C P}$-even Higgs boson in the $m_{h}^{\max }$ scenario is in agreement with the discovery of a Higgs-like state only in a relatively small strip in the $M_{A}-\tan \beta$ plane at rather low $\tan \beta$. This
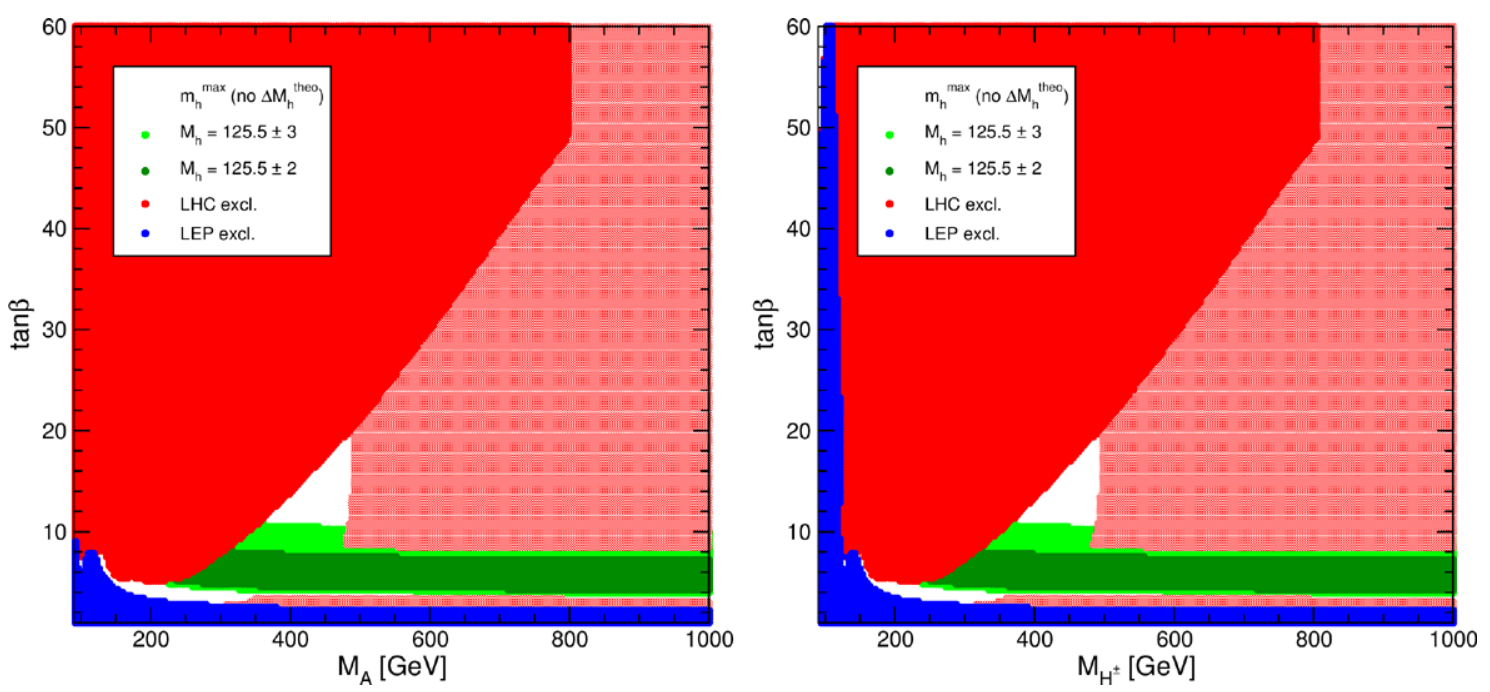

Fig. 2 The $M_{A}-\tan \beta$ (left) and $M_{H^{ \pm}-\tan \beta}$ (right) planes in the (updated) $m_{h}^{\max }$ scenario, as shown in Fig. 1 (using the same color coding), but without taking into account a theory uncertainty in the $M_{h}$ calculation of $3 \mathrm{GeV}$ in the evaluation of the existing limits (Color figure online) 

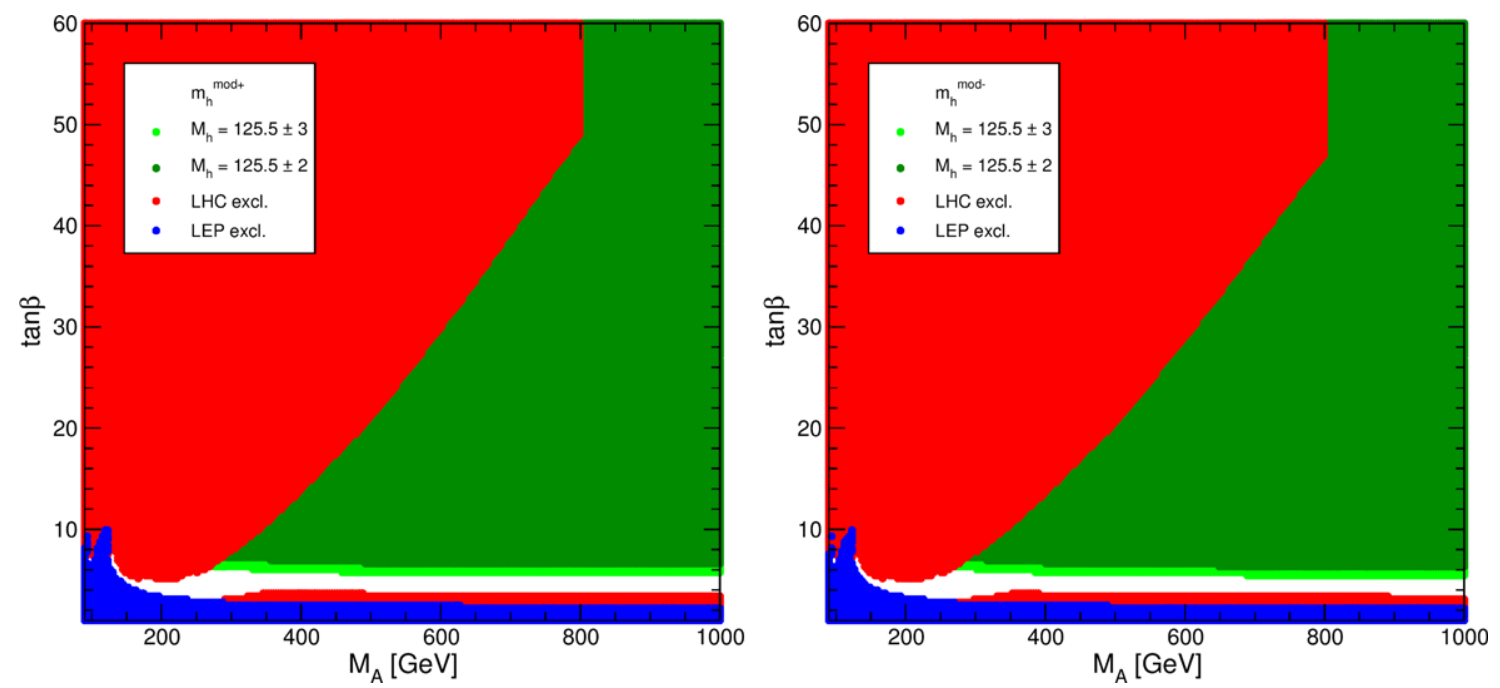

Fig. 3 The $M_{A}-\tan \beta$ plane in the $m_{h}^{\bmod +}$ (left) and $m_{h}^{\bmod -}$ (right) scenarios. The colors show exclusion regions from LEP (blue) and the LHC (red), and the favored region $M_{h}=125.5 \pm 2$ (3) $\mathrm{GeV}$ (green), see the text for details (Color figure online)

was caused by the fact that the $m_{h}^{\max }$ scenario was designed to maximize the value of $M_{h}$, so that in the decoupling region this scenario yields $M_{h}$ values that are higher than the observed mass of the signal. Departing from the parameter configuration that maximizes $M_{h}$, one naturally finds scenarios where in the decoupling region the value of $M_{h}$ is close to the observed mass of the signal over a wide region of the parameter space. A convenient way of modifying the $m_{h}^{\max }$ scenario in this way is to reduce the amount of mixing in the stop sector, i.e. to reduce $\left|X_{t} / M_{\text {SUSY }}\right|$ compared to the value of $\approx 2$ (FD calculation) that gives rise to the largest positive contribution to $M_{h}$ from the radiative corrections. This can be done for both signs of $X_{t}$.

Accordingly, we propose an " $m_{h}^{\text {mod }}$ scenario" which is a modification of the $m_{h}^{\max }$ scenario consisting of a reduction of $\left|X_{t} / M_{\text {SUSY }}\right|$. We define two variants of this scenario, the $m_{h}^{\bmod +}$ and the $m_{h}^{\bmod -}$ scenario, which differ by their sign (and absolute value) of $X_{t} / M_{\text {SUSY }}$. While the positive sign of the product $\left(\mu M_{2}\right)$ results in general in better agreement with the $(g-2)_{\mu}$ experimental results, the negative sign of the product $\left(\mu A_{t}\right)$ yields in general (assuming minimal flavor violation) better agreement with the $\mathrm{BR}(b \rightarrow s \gamma)$ measurements (see Ref. [107] for a recent analysis of the impact of other rare $B$ decay observables, most notably $B_{s} \rightarrow \mu^{+} \mu^{-}$). The parameter settings for these two scenarios are:

$$
m_{h}^{\bmod +}:
$$

$m_{t}=173.2 \mathrm{GeV}$,

$M_{\mathrm{SUSY}}=1000 \mathrm{GeV}$,

$\mu=200 \mathrm{GeV}$,

$M_{2}=200 \mathrm{GeV}$,
$X_{t}^{\mathrm{OS}}=1.5 M_{\mathrm{SUSY}} \quad$ (FD calculation),

$X_{t}^{\overline{\mathrm{MS}}}=1.6 M_{\mathrm{SUSY}} \quad(\mathrm{RG}$ calculation $)$,

$A_{b}=A_{\tau}=A_{t}$,

$m_{\tilde{g}}=1500 \mathrm{GeV}$,

$M_{\tilde{l}_{3}}=1000 \mathrm{GeV}$.

$m_{h}^{\bmod -}:$

$m_{t}=173.2 \mathrm{GeV}$,

$M_{\text {SUSY }}=1000 \mathrm{GeV}$,

$\mu=200 \mathrm{GeV}$,

$M_{2}=200 \mathrm{GeV}$,

$X_{t}^{\mathrm{OS}}=-1.9 M_{\mathrm{SUSY}} \quad$ (FD calculation),

$X_{t}^{\overline{\mathrm{MS}}}=-2.2 M_{\mathrm{SUSY}} \quad$ (RG calculation),

$A_{b}=A_{\tau}=A_{t}$,

$m_{\tilde{g}}=1500 \mathrm{GeV}$,

$M_{\tilde{l}_{3}}=1000 \mathrm{GeV}$.

Figure 3 shows the bounds on the $M_{A}-\tan \beta$ parameter space in the $m_{h}^{\text {mod+ }}$ (left) and $m_{h}^{\text {mod- }}$ (right) scenarios, using the same choice of colors as in the $m_{h}^{\max }$ scenario presented in the previous section, but from here on we show the full LHC exclusion region as solid red only. ${ }^{4}$ As anticipated, there is a large region of parameter space at moderate and large values of $\tan \beta$ where the mass of the light $\mathcal{C} \mathcal{P}$-even

\footnotetext{
${ }^{4}$ The light red color in Fig. 4 has a different meaning.
} 

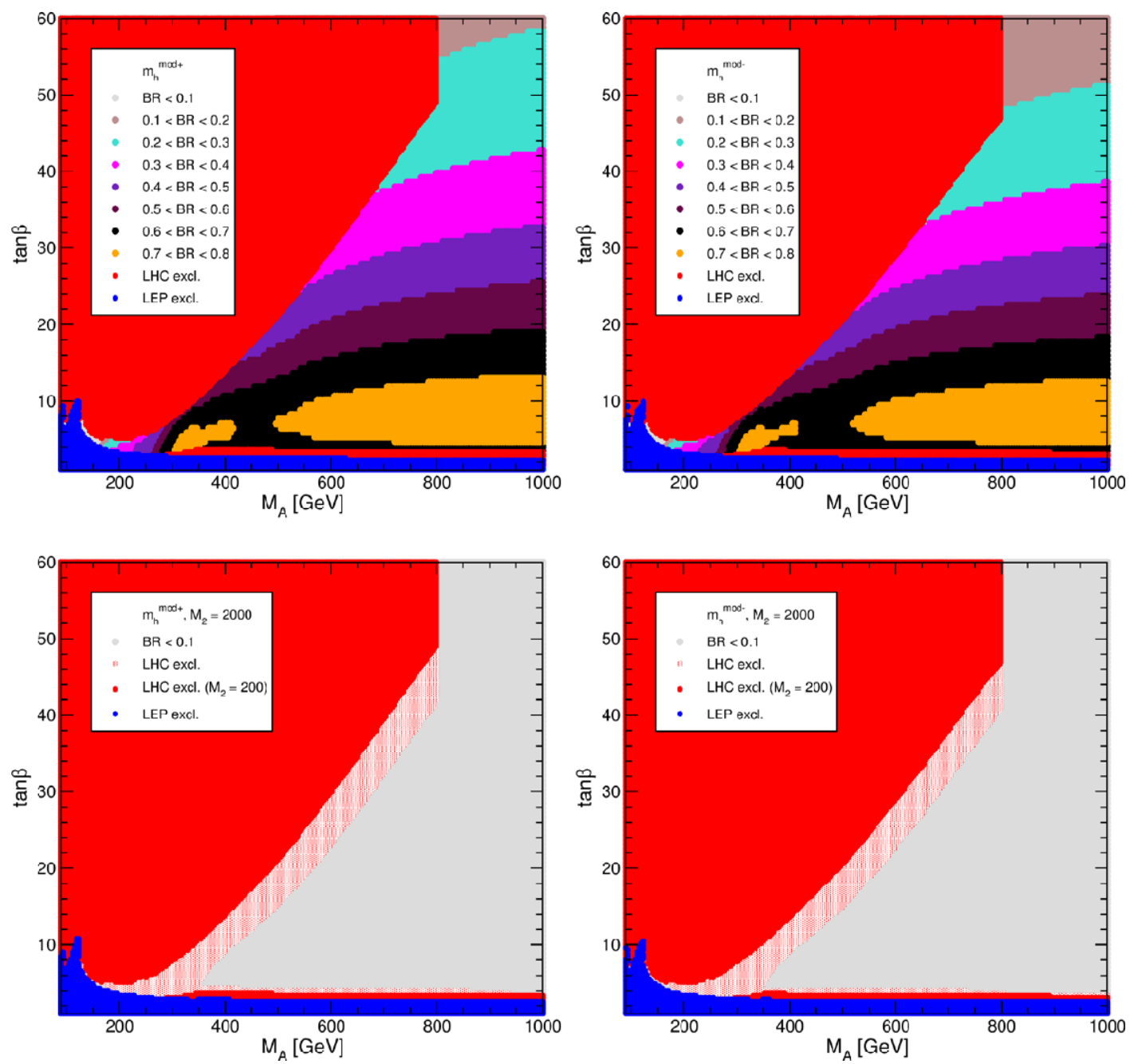

Fig. 4 Upper row: The $M_{A}-\tan \beta$ plane in the $m_{h}^{\bmod +}$ (left) and the $m_{h}^{\text {mod- }}$ scenario (right). The exclusion regions are shown as in Fig. 3, while the color coding in the allowed region indicates the average total branching ratio of $H$ and $A$ into charginos and neutralinos. In the lower row $M_{2}=2000 \mathrm{GeV}$ is used, and the color coding for the branching

Higgs boson is in good agreement with the mass value of the particle recently discovered at the LHC. Accordingly, the green area indicating the favored region now extends over almost the whole allowed parameter space of this scenario, with the exception of a small region at low values of $\tan \beta$. From Fig. 3 one can see that once the magnitude of $X_{t}$ has been changed in order to bring the mass of the light $\mathcal{C P}$ even Higgs boson into agreement with the observed mass of the signal, the change of sign of this parameter has a minor impact on the excluded regions.

As mentioned above, the exclusion limits obtained from the searches for heavy MSSM Higgs bosons in the $\tau^{+} \tau^{-}$ and $b \bar{b}$ final states are significantly affected in parameter regions where additional decay modes of the heavy MSSM Higgs bosons are open. In particular, the branching ratios for the decay of $H$ and $A$ into charginos and neutralinos

ratios of $H$ and $A$ into charginos and neutralinos is as in the upper row. The regions excluded by the LHC searches are shown in light red in these plots. For comparison, the excluded regions for the case $M_{2}=200 \mathrm{GeV}$ (as given in the plots in the upper row) is overlaid (solid red) (Color figure online)

may become large at small or moderate values of $\tan \beta$, leading to a corresponding reduction of the branching ratios into $\tau^{+} \tau^{-}$and $b \bar{b}$. In Fig. 4 we show again the $m_{h}^{\bmod +}$ (left) and $m_{h}^{\text {mod- }}$ (right) scenarios, where the excluded regions from the Higgs searches at LEP and the LHC are as before. In the upper row of Fig. 4 the color coding for the allowed region of the parameter space indicates the average value of the branching ratios for the decay of $H$ and $A$ into charginos and neutralinos (summed over all contributing final states). ${ }^{5}$ One can see from the plots that as a consequence of the relatively low values of $\mu$ and $M_{2}$ in this benchmark scenario decays of $H$ and $A$ into charginos and neutralinos are kine-

${ }^{5}$ The branching ratios into charginos and neutralinos turn out to be very similar for the heavy $\mathcal{C P}$-even Higgs boson, $H$, and the $\mathcal{C P}$-odd Higgs boson, $A$, in this region of parameter space. 


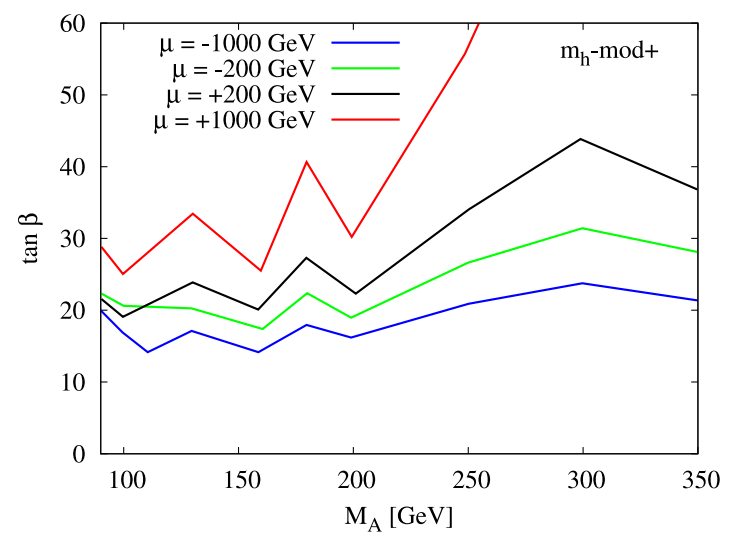

Fig. 5 Exclusion limits from the most recent CMS analysis of the channel $b \bar{b} \phi, \phi \rightarrow b \bar{b}$ (with $\phi=h, H, A$ ) [108] are presented in the

matically open essentially in the whole allowed parameter space of the scenario, with the exception of a small region with rather small $M_{A}$. The branching ratios for the decays of $H$ and $A$ into charginos and neutralinos reach values in excess of $70 \%$ for small and moderate values of $\tan \beta$.

The impact of the corresponding reduction of the branching ratios of $H, A$ into $\tau^{+} \tau^{-}$and $b \bar{b}$ on the excluded region can be read off from the plots in the lower row of Fig. 4. In those plots we have set $M_{2}=2000 \mathrm{GeV}$, which suppresses the decays of $H$ and $A$ into charginos and neutralinos. The region excluded by the LHC searches for MSSM Higgs bosons is shown in light red for this case. Overlaid for comparison is the excluded region obtained for $M_{2}=200 \mathrm{GeV}$, as given by the plots in the upper row (solid red). One can see that the impact of the decays into charginos and neutralinos on the excluded region in the $M_{A}-\tan \beta$ plane is sizable, amounting typically to a shift in the excluded value for $\tan \beta$ by more than $\Delta \tan \beta=5$ for a given value of $M_{A}$.

As mentioned above, another decay mode that is kinematically possible over a large part of the parameter space of the $m_{h}^{\text {mod }}$ scenarios is the decay rate of $H \rightarrow h h$. For $M_{2}=200 \mathrm{GeV}$ (plots in the upper row of Fig. 4) and $M_{A}=300 \mathrm{GeV}$ we find in the $m_{h}^{\bmod +}\left(m_{h}^{\bmod -}\right)$ scenario $\mathrm{BR}(H \rightarrow h h)=12 \%(11 \%)$ for $\tan \beta=7$ and $\mathrm{BR}(H \rightarrow$ $h h)=17 \%(16 \%)$ for $\tan \beta=6$. Increasing $M_{2}$ to $M_{2}=$ $2000 \mathrm{GeV}$ (plots in the lower row of Fig. 4) suppresses the decays into charginos and neutralinos, and correspondingly enhances the decay $H \rightarrow h h$. For $M_{A}=300 \mathrm{GeV}$ in the $m_{h}^{\bmod +}\left(m_{h}^{\bmod -}\right)$ scenario we obtain $\operatorname{BR}(H \rightarrow h h)=19 \%$ $(18 \%)$ for $\tan \beta=7$ and $\operatorname{BR}(H \rightarrow h h)=29 \%$ (27\%) for $\tan \beta=6$. As already mentioned, we encourage ATLAS and CMS to enhance the sensitivity of their searches for MSSM Higgs bosons by performing also dedicated searches for Higgs decays into SUSY particles and into a pair of lighter Higgs bosons.

For the benchmarks proposed in this paper a certain value for the parameter $\mu$ is specified. However, we suggest to

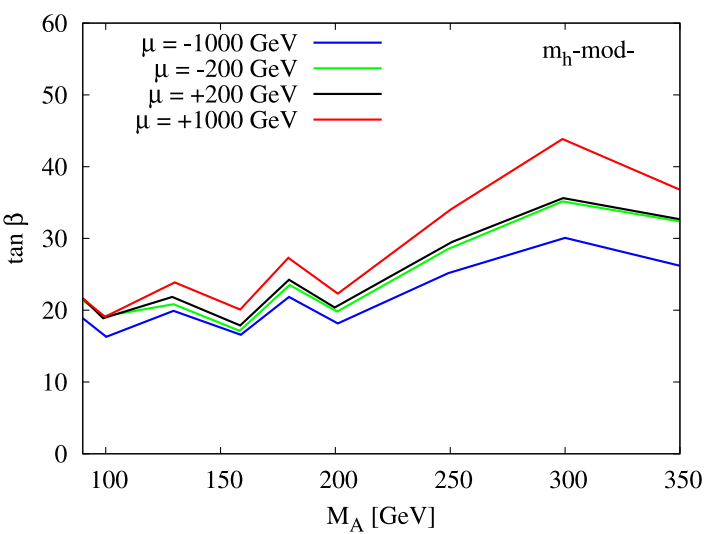

$M_{A}-\tan \beta$ plane for the scenarios $m_{h}^{\bmod +}$ (left) and $m_{h}^{\bmod -}$ (right) with variation of the $\mu$ parameter as indicated by the legend

investigate the impact of an enhancement or suppression of the bottom Yukawa coupling by varying the parameter $\mu$ according to Eq. (19). For the Higgs decays into $\tau^{+} \tau^{-}$, see Eq. (1), a partial cancelation of the associated $\Delta_{b}$ corrections occurs between the contributions to the production and the decay, leading to a relatively mild dependence on the bottom Yukawa coupling and therefore on $\Delta_{b}$ [44]. On the other hand, for the associated production and decay into bottom quarks, see Eq. (2), the $\Delta_{b}$ corrections enter in a similar way for the production and decay part, so that their overall effect is significantly larger, leading to a more pronounced dependence on the sign and size of the $\mu$ parameter [44]. Negative values of $\mu$ lead to a stronger bottomquark Yukawa coupling and therefore a larger production rate and a larger parameter range exclusion. The bounds on the parameter space from this channel tend to be weaker than those from $\tau \tau$ searches, and they are therefore not explicitly visible in Fig. 3. In order to display the effect of the corrections to the bottom Yukawa coupling we focus now explicitly on the channel $b \bar{b} \phi, \phi \rightarrow b \bar{b}$, where $\phi=h, H, A$. Using the latest result from CMS for this channel [108], Fig. 5 shows the reach in the $M_{A}-\tan \beta$ plane of the $m_{h}^{\bmod +}$ (left) and $m_{h}^{\text {mod- }}$ (right) scenarios for $\mu= \pm 200 \mathrm{GeV}$, $\pm 1000 \mathrm{GeV}$ (see also [109]). ${ }^{6}$ In the $m_{h}^{\bmod +}$ scenario one can observe a very large variation with the sign and absolute value of $\mu$. For example, for $M_{A}=250 \mathrm{GeV}$ one finds for $\mu=-1000 \mathrm{GeV}$ an exclusion in $\tan \beta$ down to about $\tan \beta=20$, while for the reversed sign of $\mu$ the excluded region starts only above $\tan \beta=50$. The dependence on $\mu$ is less pronounced in the $m_{h}^{\bmod -}$ scenario, i.e. for negative values of $X_{t}$, which is a consequence of a partial compensation between the main contributions to $\Delta_{b}$, see Eq. (14).

\footnotetext{
${ }^{6}$ We have verified our implementation of this limit against the results from CMS [108], which are given for the (original) $m_{h}^{\max }$ scenario with $\mu= \pm 200 \mathrm{GeV}$. The "zig-zag"-type variation of the bounds originates from the original bounds in Ref. [108].
} 


\subsection{The light stop scenario}

The measured value of the lightest $\mathcal{C P}$-even Higgs mass of about $125.5 \mathrm{GeV}$ may only be achieved in the MSSM by relatively large radiative contributions from the top-stop sector. It is well known that this can only be obtained if the mixing parameter $X_{t}$ in the stop sector is larger than the average stop mass. The dependence of $M_{h}$ on the stop mass scale is logarithmic and allows for values of $M_{\text {SUSY }}$ below the TeV scale. Values of $M_{\text {SUSY }}$ significantly below the TeV scale are still possible if $X_{t}$ is close to the value that maximizes the lightest $\mathcal{C P}$-even Higgs mass (or, to a lesser extent, close to the maximum for negative values of $X_{t}$ ). Such a large value of $\left|X_{t}\right|$ and a relatively low value of $M_{\text {SUSY }}$ necessarily lead to the presence of a light stop. Such a light stop may be searched for in direct production at the LHC, but has also a relevant impact on the lightest $\mathcal{C P}$-even Higgs production rates. In particular, a light stop may lead to a relevant modification of the gluon fusion rate $[42,110]$.

The contribution of light stops to the gluon fusion amplitude may be parametrized in terms of the physical stop masses and the mixing parameter. Making use of low-energy theorems $[111,112]$ it is easy to see that the stops give rise to an additional contribution to the gluon fusion amplitude which is approximately given by [113-115]

$\delta \mathcal{A}_{h g g} / \mathcal{A}_{h g g}^{\mathrm{SM}} \simeq \frac{m_{t}^{2}}{4 m_{\tilde{t}_{1}}^{2} m_{\tilde{t}_{2}}^{2}}\left(m_{\tilde{t}_{1}}^{2}+m_{\tilde{t}_{2}}^{2}-X_{t}^{2}\right)$

where $\mathcal{A}_{h g g}^{\mathrm{SM}}$ denotes the gluon fusion amplitude in the SM. Values of $X_{t}$ in the range $2 M_{\text {SUSY }} \lesssim X_{t} \lesssim 2.5 M_{\text {SUSY }}$ then lead to negative contributions to this amplitude and to reduced values of the gluon fusion rate. We propose a light stop scenario with the following parameters,

light stop:

$m_{t}=173.2 \mathrm{GeV}$,

$M_{\text {SUSY }}=500 \mathrm{GeV}$,

$\mu=350 \mathrm{GeV}$,

$M_{2}=350 \mathrm{GeV}$,

$X_{t}^{\mathrm{OS}}=2.0 M_{\mathrm{SUSY}} \quad$ (FD calculation $)$,

$X_{t}^{\overline{\mathrm{MS}}}=2.2 M_{\mathrm{SUSY}} \quad$ (RG calculation)

$A_{b}=A_{t}=A_{\tau}$,

$m_{\tilde{g}}=1500 \mathrm{GeV}$,

$M_{\tilde{l}_{3}}=1000 \mathrm{GeV}$.

These parameters lead to a lighter stop and a heavier stop mass of about $325 \mathrm{GeV}$ and $670 \mathrm{GeV}$, respectively, and a negative correction of the gluon fusion amplitude of about
$8 \%$. The light stop scenario can be regarded as an update of the gluophobic Higgs scenario defined in Ref. [42].

The values of $\mu$ and $M_{2}$ in the light stop scenario have been chosen to be in agreement with the current exclusion bounds on direct light stop production at the LHC [116127]. ${ }^{7}$ The two-body decay modes that are kinematically open are $\tilde{t}_{1} \rightarrow b \tilde{\chi}_{1}^{+}$and $\tilde{t}_{1} \rightarrow c \tilde{\chi}_{1}^{0}$ with $m_{\tilde{\chi}_{1}^{ \pm}} \approx 295 \mathrm{GeV}$ and $m_{\tilde{\chi}_{1}^{0}} \approx 163 \mathrm{GeV}$. The first decay results in very soft decay products. While the latter decay is expected to be suppressed in minimal flavor violating schemes, it could in general be sizable. Analyses have been performed at the Tevatron [128, 129]; however, currently there are no dedicated LHC searches in this channel. If this channel turned out to be relevant, due to its difficult final state it would pose a challenge to the experimental analyses.

There is also a correction to the diphoton amplitude, but since in the diphoton case the dominant SM contribution comes from $W$ loops, which are of opposite sign and about a factor 4 larger than the top contributions, the stop contributions lead to only a small modification, smaller than about $3 \%$, of this amplitude.

Figure 6 shows the $M_{A}-\tan \beta$ plane in the light stop scenario, as well as a comparison of the gluon fusion rates for $h$ production to those obtained in the SM. For this comparison, we define the quantity

$r_{g g}=\frac{\Gamma(h \rightarrow g g)_{\mathrm{MSSM}}}{\Gamma(h \rightarrow g g)_{\mathrm{SM}}}$,

which gives a rough approximation of the relative suppres-

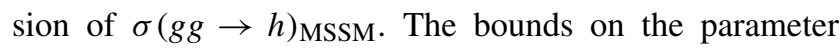
space (as before obtained with HiggsBounds) are similar to the ones obtained in the $m_{h}^{\bmod }$ scenarios. However, the gluon fusion rate is between $10 \%$ and $15 \%$ lower than in the $\mathrm{SM}$, as expected from Eq. (23). This shift is similar in magnitude to the current theoretical uncertainties on the gluon fusion cross section from e.g. the strong coupling constant and parton distribution functions.

\subsection{The light stau scenario}

While light stops may lead to a large modification of the gluon fusion rate, with a relative minor effect on the diphoton rate, it has been shown that light staus, in the presence of large mixing, may lead to important modifications of the diphoton decay width of the lightest $\mathcal{C P}$-even Higgs boson,

\footnotetext{
${ }^{7}$ The values of $\mu, M_{1}$ and $M_{2}$ could be adjusted to slightly larger values if the currently proposed values were excluded by future experiments. For instance, the choice $M_{1}=350 \mathrm{GeV}, M_{2}=\mu=400 \mathrm{GeV}$ leads to a SUSY spectrum that is very difficult to test at the LHC. In general, for a given value of $\tan \beta$ and $M_{A}$, slightly larger values of $\mu$ and $M_{1,2}$ would lead to a small decrease of the value of $M_{h}$ and therefore to a small shift of the green areas to larger values of $\tan \beta$.
} 

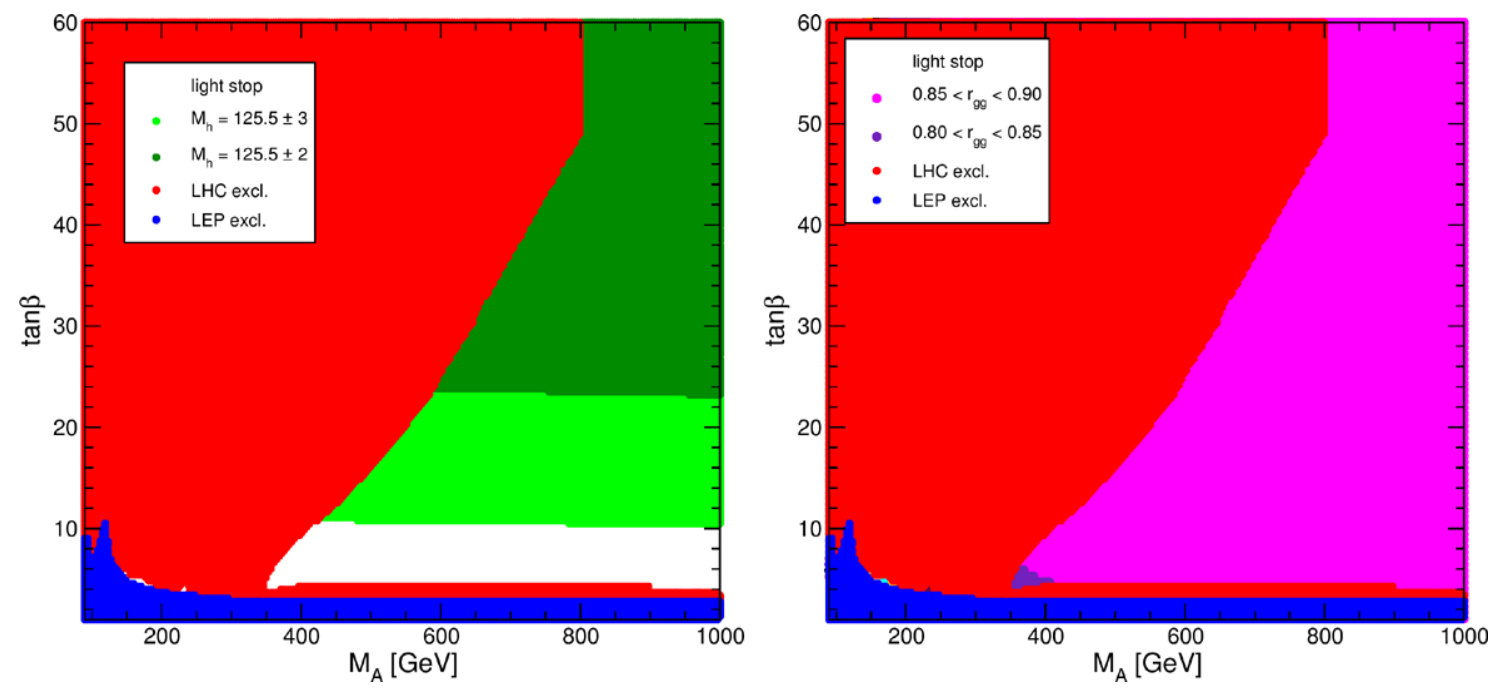

Fig. 6 The $M_{A}-\tan \beta$ plane in the light stop scenario; left: with the same color coding as in Fig. 3; right: the resulting suppression of the gluon fusion rate, as indicated by the legend (Color figure online)

$\Gamma(h \rightarrow \gamma \gamma)$ [16-19, 130-133]. Large mixing in the stau sector may happen naturally for large values of $\tan \beta$, for which the mixing parameter $X_{\tau}=A_{\tau}-\mu \tan \beta$ becomes large. Similarly to the modifications of the gluon fusion rate in the light stop scenario, one can use the low-energy Higgs theorems [111, 112] to obtain the modifications of the decay rate of the Higgs boson to photon pairs. The correction to the amplitude of Higgs decays to diphotons is approximately given by [16-19, 113-115]

$\delta \mathcal{A}_{h \gamma \gamma} / \mathcal{A}_{h \gamma \gamma}^{\mathrm{SM}} \simeq-\frac{2 m_{\tau}^{2}}{39 m_{\tilde{\tau}_{1}}^{2} m_{\tilde{\tau}_{2}}^{2}}\left(m_{\tilde{\tau}_{1}}^{2}+m_{\tilde{\tau}_{2}}^{2}-X_{\tau}^{2}\right)$,

where $\mathcal{A}_{h \gamma \gamma}^{\mathrm{SM}}$ denotes the diphoton amplitude in the SM.

Due to the large $\tan \beta$ enhancement $X_{\tau}$ is naturally much larger than the stau masses and hence the corrections are positive and become significant for large values of $\tan \beta$. As stressed above, the current central value of the measured diphoton rate of the state discovered at the LHC is somewhat larger than the expectations for a SM Higgs, which adds motivation for investigating the phenomenology of a scenario with an enhanced diphoton rate. We therefore propose a light stau scenario. In the definition of the parameters we distinguish the cases whether or not $\tau$ mass threshold corrections, $\Delta_{\tau}$, are incorporated in the computation of the stau spectrum (this is the case in CPsuperH, but not in the present version of FeynHiggs). We mark the case where those corrections are included as " $\left(\Delta_{\tau}\right.$ calculation $)$ ". We define the parameters of the light stau scenario as follows:

light stau:

$m_{t}=173.2 \mathrm{GeV}$,

$M_{\mathrm{SUSY}}=1000 \mathrm{GeV}$, $\mu=500 \mathrm{GeV}$,

$\mu=450 \mathrm{GeV} \quad\left(\Delta_{\tau}\right.$ calculation $)$,

$M_{2}=200 \mathrm{GeV}$,

$M_{2}=400 \mathrm{GeV} \quad\left(\Delta_{\tau}\right.$ calculation $)$,

$X_{t}^{\mathrm{OS}}=1.6 M_{\mathrm{SUSY}} \quad($ FD calculation $)$,

$X_{t}^{\overline{\mathrm{MS}}}=1.7 M_{\mathrm{SUSY}} \quad$ (RG calculation),

$A_{b}=A_{t}$,

$A_{\tau}=0$,

$m_{\tilde{g}}=1500 \mathrm{GeV}$,

$M_{\tilde{l}_{3}}=245 \mathrm{GeV}$,

$M_{\tilde{l}_{3}}=250 \mathrm{GeV} \quad\left(\Delta_{\tau}\right.$ calculation $)$.

Figure 7 shows the $M_{A}-\tan \beta$ plane in the light stau scenario (left), as well as comparison of the $h \rightarrow \gamma \gamma$ width to the SM case (right). Concerning the exclusion bounds from the Higgs searches at LEP and the LHC, the main difference with respect to the $m_{h}^{\bmod }$ scenarios is present at low values of $\tan \beta$, where the LHC exclusion in the light stau scenario is somewhat stronger. This results from a suppression of the decays into charginos and neutralinos caused by the relatively large (default) value of $\mu$ in the light stau scenario. The right panel shows the enhancement of the diphoton decay rate of the lightest $\mathcal{C P}$-even Higgs boson with respect to the SM (with $r_{\gamma \gamma}$ defined analogously to $r_{g g}$ in Eq. (25)). As expected, a significant enhancement is present at large values of $\tan \beta>50$, for which the lightest stau approaches a mass of about $100 \mathrm{GeV}$, close to the LEP limit for the stau mass [91]. For non-zero values of $A_{\tau}$ in this scenario, 

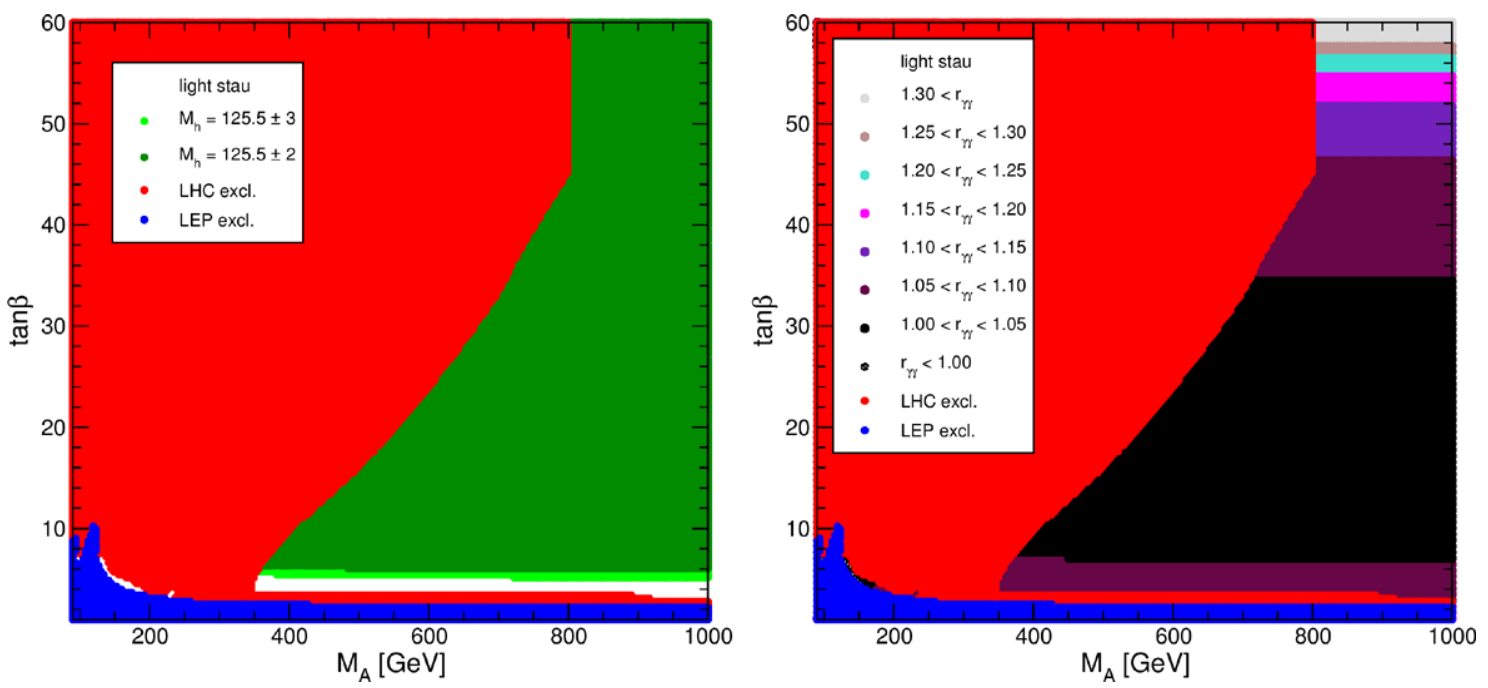

Fig. 7 Left: The $M_{A}-\tan \beta$ plane in the light stau scenario, with the same color coding as in Fig. 3. Right: The effect of light staus on the decay rate $h \rightarrow \gamma \gamma$, where the quantity $r_{\gamma \gamma}$ is defined in analogy to $r_{g g}$ in Eq. (25) (Color figure online)

the coupling of the down-type fermions to the lightest Higgs boson may be modified [16-19]. The decay rate of $H / A$ into staus can also become sizable, see the discussion in Sect. 3.5.

\subsection{The $\tau$-phobic Higgs scenario}

Besides the loop effects on the Higgs vertices described in the previous sections, also propagator-type corrections involving the mixing between the two $\mathcal{C P}$-even Higgs bosons of the MSSM can have an important impact. In particular, this type of corrections can lead to relevant modifications of the Higgs couplings to down-type fermions, which can approximately be taken into account via an effective mixing angle $\alpha_{\text {eff }}$ (see Ref. [134, 135]). This modification occurs for large values of the $A_{t, b, \tau}$ parameters and large values of $\mu$ and $\tan \beta .^{8}$

The scenario that we propose can be regarded as an update of the small $\alpha_{\text {eff }}$ scenario proposed in Ref. [42]. The parameters are:

$\underline{\tau-\text { phobic Higgs: }}$

$m_{t}=173.2 \mathrm{GeV}$,

$M_{\mathrm{SUSY}}=1500 \mathrm{GeV}$,

$\mu=2000 \mathrm{GeV}$,

$M_{2}=200 \mathrm{GeV}$,

\footnotetext{
${ }^{8}$ Large values of $A_{t, b, \tau}$ and $\mu$ are in principle constrained by the requirement that no charge and color breaking minima should appear in the potential [136-138], or at least that there is a sufficiently long-lived meta-stable vacuum. However, a detailed analysis of this issue is beyond the scope of this paper, and we leave it for a future analysis.
}

$X_{t}^{\mathrm{OS}}=2.45 M_{\mathrm{SUSY}} \quad($ FD calculation $)$,

$X_{t}^{\overline{\mathrm{MS}}}=2.9 M_{\mathrm{SUSY}} \quad$ (RG calculation),

$A_{b}=A_{t}$,

$A_{\tau}=0$,

$m_{\tilde{g}}=1500 \mathrm{GeV}$,

$M_{\tilde{l}_{3}}=500 \mathrm{GeV}$.

The relatively low value of $M_{\tilde{l}_{3}}=500 \mathrm{GeV}$ and the large value of $\mu$ give rise to rather light staus also in the $\tau$-phobic Higgs scenario, in particular in the region of large $\tan \beta$. The corrections from the stau sector have an important influence on the Higgs couplings to down-type fermions in this scenario. Furthermore, in this scenario decays of the heavy $\mathcal{C P}$ even Higgs boson into light staus, $H \rightarrow \tilde{\tau}_{1}^{+} \tilde{\tau}_{1}^{-}$, occur with a large branching fraction in the region of large $\tan \beta$ and sufficiently high $M_{A}$. For example, for $M_{A}=800 \mathrm{GeV}$ and $\tan \beta=45$, we obtain $\operatorname{BR}\left(H \rightarrow \tilde{\tau}_{1}^{+} \tilde{\tau}_{1}^{-}\right)=67 \%$.

Figure 8 shows the bounds on the $M_{A}-\tan \beta$ parameter space in the $\tau$-phobic Higgs scenario. As in the light stau scenario, the most important modification with respect to the $m_{h}^{\text {mod }}$ scenarios is a larger exclusion at low values of $\tan \beta$ induced by a decrease of the decay rate into charginos and neutralinos.

Figure 9 shows the modification of the decay rate for the lightest $\mathcal{C P}$-even Higgs boson into bottom quarks $\left(r_{b b}\right)$ and $\tau$-leptons $\left(r_{\tau \tau}\right)$, both defined analogously to $r_{g g}$, see Eq. (25). The variations are most important at large values of $\tan \beta$, and they increase for smaller values of $M_{A}$, where the LHC exclusion limit from MSSM Higgs searches becomes very significant. Still, as can be seen from the figure, modifications of the partial Higgs decay width into $\tau^{+} \tau^{-}$ larger than $20 \%$, and of the decay width into bottom quarks larger than $10 \%$ may occur within this scenario. 


\subsection{The low- $M_{H}$ scenario}

As it was pointed out in Refs. [15, 20, 21, 35, 36], besides the interpretation of the Higgs-like state at $\sim 125.5 \mathrm{GeV}$ in terms of the light $\mathcal{C P}$-even Higgs boson of the MSSM it is also possible, at least in principle, to identify the observed signal with the heavy $\mathcal{C P}$-even Higgs boson of the MSSM. In this case the Higgs sector would be very different from the SM case, since all five MSSM Higgs bosons would be light. The heavy $\mathcal{C P}$-even Higgs boson would have a mass around $125.5 \mathrm{GeV}$ and behave roughly SM-like, while the light $\mathcal{C} \mathcal{P}$-even Higgs boson of the MSSM would have heavily suppressed couplings to gauge bosons. Due to the rather spectacular phenomenology of such a scenario, the available

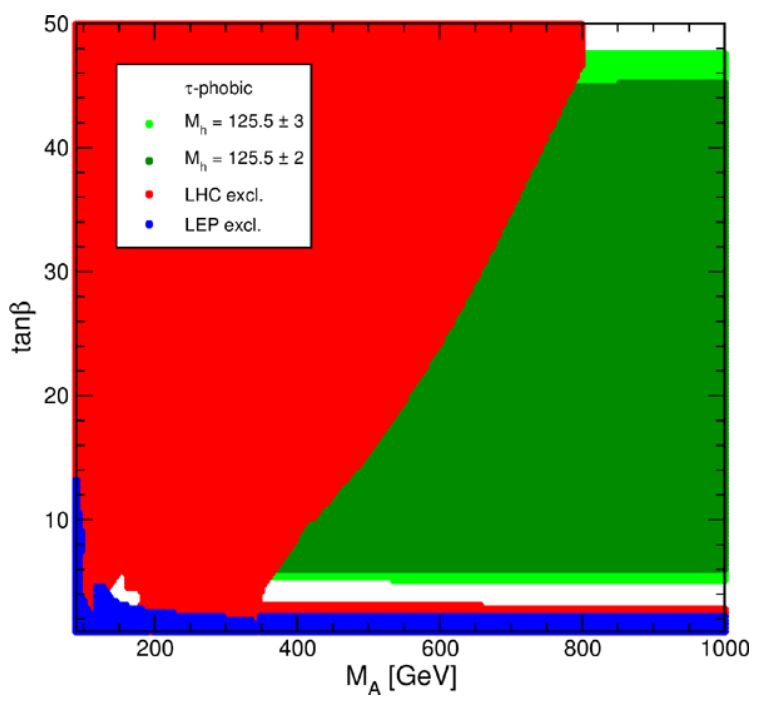

Fig. 8 The $M_{A}-\tan \beta$ plane in the $\tau$-phobic Higgs scenario. The color coding is the same as in Fig. 3 (Color figure online)

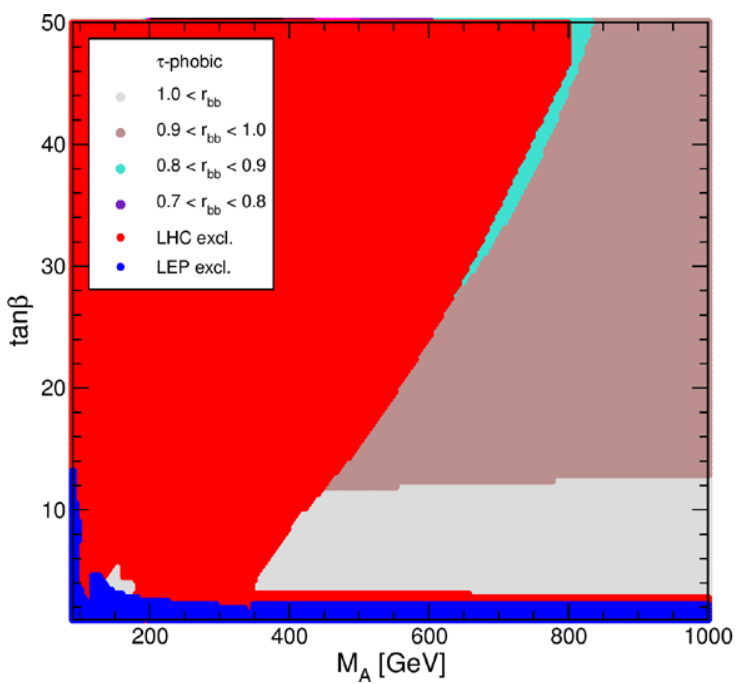

parameter space is already affected by existing search limits, and the prospects for discovering a non-SM-like Higgs in the near future would be very good.

The most relevant limits probing such a scenario at present arise from the searches for MSSM Higgs bosons in the $g g, b \bar{b} \rightarrow h, H, A \rightarrow \tau \tau$ channel, but also the search for a light charged Higgs in top-quark decays has an interesting sensitivity. The results for the $g g, b \bar{b} \rightarrow h, H, A \rightarrow \tau \tau$ channel have recently been updated by CMS [98]. However, it is difficult to assess the impact of those new results on the viability of such a scenario, since they have been presented only for the $m_{h}^{\max }$ scenario (i.e., no cross section limits have been provided which could readily be applied to other scenarios; an attempt to incorporate a rough estimate of the new CMS result has been made in Higgs Bounds 4 . 0 . 0 [94-97], which we have used for producing the plots in this paper). Besides Higgs search limits also limits from flavor physics can place relevant constraints on this kind of scenario. It was found in Refs. [20, 21, 26, 27] that flavor constraints could lead to tension with the allowed parameter space (which might be alleviated by taking into account some Non-Minimal Flavor Violation [139]). We do not take these indirect constraints into account in this analysis. In view of the rich and interesting phenomenology, we include a scenario of this kind among the benchmarks that we propose. In particular, this scenario could provide a useful benchmark for the ongoing charged Higgs boson searches in the MSSM.

In this scenario we deviate from the definition of an $M_{A^{-}}$ $\tan \beta$ plane, since it is clear that a relatively small value of $M_{A}$ (and correspondingly $M_{H^{ \pm}}$) is required. $M_{A}$ is therefore fixed to $M_{A}=110 \mathrm{GeV}$ (other choices for $M_{A}$ in this low-mass region would also be possible), and instead $\mu$ is

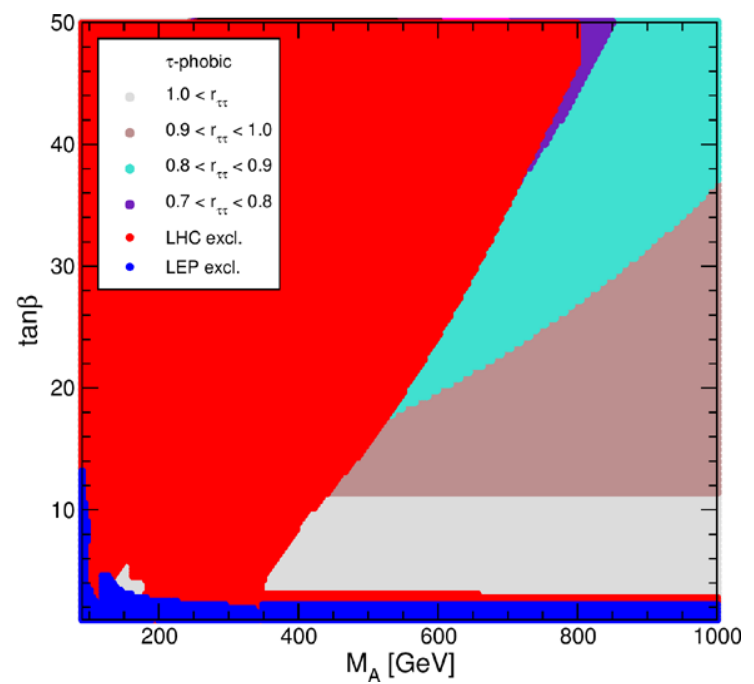

Fig. 9 Modification of the decay rate for the lightest $\mathcal{C P}$-even Higgs boson into bottom quarks $\left(r_{b b}\right.$, left $)$ and $\tau$-leptons $\left(r_{\tau \tau}\right.$, right $)$ in the $\tau$-phobic Higgs scenario, where $r_{b b}$ and $r_{\tau \tau}$ are defined in analogy to $r_{g g}$ in Eq. (25) 
varied. Otherwise we choose the same parameters as for the $\tau$-phobic Higgs scenario, with the exception that we set $M_{\tilde{l}_{3}}=1000 \mathrm{GeV}$, while the value in the $\tau$-phobic Higgs scenario is $M_{\tilde{l}_{3}}=500 \mathrm{GeV}$ (see the discussion above). Accordingly, the parameters proposed for this scenario are: ${ }^{9}$

$$
\underline{\text { low }-M_{H} \text { : }}
$$

$m_{t}=173.2 \mathrm{GeV}$,

$M_{A}=110 \mathrm{GeV}$,

$M_{\mathrm{SUSY}}=1500 \mathrm{GeV}$,

$M_{2}=200 \mathrm{GeV}$,

$X_{t}^{\mathrm{OS}}=2.45 M_{\text {SUSY }} \quad($ FD calculation $)$,

$X_{t}^{\overline{\mathrm{MS}}}=2.9 M_{\mathrm{SUSY}} \quad(\mathrm{RG}$ calculation $)$,

$A_{b}=A_{\tau}=A_{t}$,

$m_{\tilde{g}}=1500 \mathrm{GeV}$,

$M_{\tilde{l}_{3}}=1000 \mathrm{GeV}$.

Instead of $M_{A}$ one can also use $M_{H^{ \pm}}$as input parameter, as is done, e.g., in CPsuperH. In this case one should choose as input value $M_{H^{ \pm}}=132 \mathrm{GeV}$, leading to very similar phenomenology.

In Fig. 10 we show the $\mu-\tan \beta$ plane in the low- $M_{H}$ scenario. The green shades indicate the region where $M_{H}=$ $125.5 \pm 2$ (3) GeV. The yellow and black areas also have

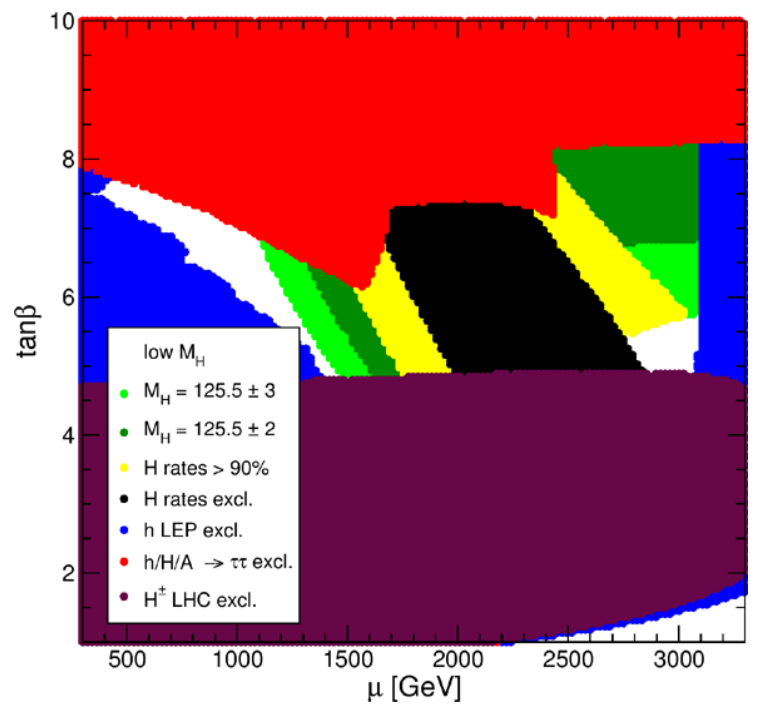

Fig. 10 Experimentally favored and excluded regions in the $\mu-\tan \beta$ plane in the low- $M_{H}$ scenario. Details of the color coding (as indicated in the legend) are described in the text (Color figure online)

\footnotetext{
${ }^{9}$ The remark made in the previous section about the constraints from charge and color breaking minima in the scalar potential applies also here.
}

$M_{H}=125.5 \pm 3 \mathrm{GeV}$, where the yellow area additionally satisfies the requirement that the rates for the $g g \rightarrow H$, $H \rightarrow \gamma \gamma$ and $H \rightarrow Z Z^{*}$ channels, as approximated by $(X=\gamma, Z)$

$R_{X X}=\frac{\Gamma(H \rightarrow g g)_{\mathrm{MSSM}} \times \mathrm{BR}(H \rightarrow X X)_{\mathrm{MSSM}}}{\Gamma(H \rightarrow g g)_{\mathrm{SM}} \times \mathrm{BR}(H \rightarrow X X)_{\mathrm{SM}}}$,

are at least at $90 \%$ of their SM value for the same Higgs mass. The black region in Fig. 10 indicates where the rates for $H$ decay to gauge bosons become too high, such that these points are excluded by HiggsBounds. As before, the blue area is excluded by LEP Higgs searches, whereas the solid red is excluded from LHC searches for the neutral MSSM Higgs bosons, $h, H$ and $A$ in the $\tau^{+} \tau^{-}$decay channel. The purple region is excluded by charged Higgs boson searches at the LHC. The white area at very large values of $\mu$ and low $\tan \beta$ is unphysical, i.e. this parameter region is theoretically inaccessible.

One can see from Fig. 10 that, as expected, such a scenario is confined to a relatively small range of $\tan \beta$ values (and, as discussed above, the same holds for $M_{A}$ ). It is interesting to note that the searches for all five MSSM Higgs bosons contribute in a significant way to the excluded regions displayed in Fig. 10. Concerning the light $\mathcal{C P}$-even Higgs boson, within the yellow region in Fig. 10 its mass turns out to be rather low, in the range $77 \mathrm{GeV} \lesssim M_{h} \lesssim$ $102 \mathrm{GeV}$, i.e. significantly below the LEP limit for a SMlike Higgs [37]. The couplings of the light $\mathcal{C P}$-even Higgs boson to gauge bosons are heavily suppressed in this region, leading to rates for the relevant cross sections that are typically smaller by a factor of $2-10$ than the LEP limits [37].

While the existing limits from the searches for the MSSM Higgs bosons constrain the parameter space of the low- $M_{H}$ scenario, according to our assessment based on HiggsBounds 4.0.0 there remains an interesting parameter region that is unexcluded, as displayed in Fig. 10. The proposed low- $M_{H}$ benchmark scenario is intended to facilitate a proper experimental analysis that will answer the question whether scenario giving rise to Higgs phenomenology that is very different from the SM case is still viable in the MSSM. As discussed above, besides the searches for neutral MSSM Higgs bosons in $\tau^{+} \tau^{-}$final states also charged Higgs searches have a high sensitivity for probing this scenario. In order to investigate the prospects for charged Higgs searches in top-quark decays in more detail, we show in Fig. 11 the predictions for $\mathrm{BR}\left(t \rightarrow H^{ \pm} b\right)$ (denoted as "BR" in the plot) in the unexcluded region of the $\mu-\tan \beta$ plane of the low- $M_{H}$ scenario. One observes that this branching ratio is just below the current experimental limits [100, 101], which are at the level of $1 \%$. 


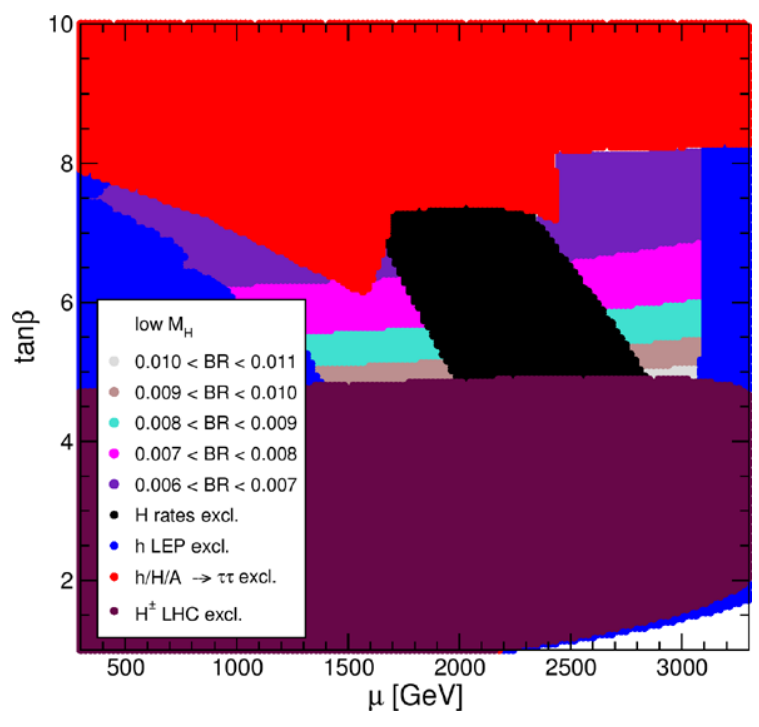

Fig. 11 Values of $\operatorname{BR}\left(t \rightarrow H^{ \pm} b\right)$ (denoted as "BR") in the $\mu-\tan \beta$ plane in the low $-M_{H}$ scenario. The experimentally excluded regions are indicated as in Fig. 10

\section{Conclusions}

In this paper we have proposed new benchmark scenarios for MSSM Higgs boson searches at the LHC. The proposed benchmarks are expressed in terms of low-energy MSSM parameters and are restricted to the $(\mathcal{C P}$-conserving $)$ case of real parameters. The benchmark scenarios take into account the recent discovery of a Higgs-like state at $\sim 125.5 \mathrm{GeV}$, i.e. over a wide range of their parameter space they are compatible with both the mass and the detected production rates of the observed signal. This refers to the interpretation of the signal in terms of the light $\mathcal{C P}$-even Higgs boson of the MSSM, with the exception of the low- $M_{H}$ scenario, where the observed signal is interpreted as the heavier $\mathcal{C P}$ even Higgs boson. For each scenario we have investigated the impact on the parameter space from the current exclusion bounds from Higgs searches at LEP, the Tevatron and the LHC (taking both experimental and theory uncertainties into account). The benchmark scenarios have been chosen to demonstrate certain features of MSSM Higgs phenomenology.

The proposed set of benchmarks comprises a slightly updated version of the well-known $m_{h}^{\max }$ scenario, which can be used to obtain conservative lower bounds on $M_{A}, M_{H^{ \pm}}$ and $\tan \beta$ via the interpretation of the light $\mathcal{C P}$-even Higgs as the newly observed state at $\sim 125.5 \mathrm{GeV}$ (including theoretical uncertainties). Furthermore we propose a modified scenario $\left(m_{h}^{\text {mod }}\right)$, which differs from the $m_{h}^{\text {max }}$ scenario by reducing the mixing in the stop sector (parametrized by $\left.\left|X_{t} / M_{\mathrm{SUSY}}\right|\right)$ compared to the value that maximizes $M_{h}$. Two versions of this scenario are proposed, one with a positive and one with a negative sign of $X_{t}$. Within (both versions of) the $m_{h}^{\text {mod }}$ scenario the light $\mathcal{C P}$-even Higgs boson can be interpreted as the newly discovered state within the whole parameter space of the $M_{A}-\tan \beta$ plane that is unexcluded by limits from Higgs searches at LEP and the LHC, except for a small region with very small values of $\tan \beta$. We expect the $m_{h}^{\text {mod }}$ scenario to be useful for the future interpretations of the searches for the heavy MSSM Higgs bosons $H, A$ and $H^{ \pm}$.

As we have discussed in some detail for the $m_{h}^{\max }$ and $m_{h}^{\text {mod }}$ scenarios, the searches for the heavy MSSM Higgs bosons $H$ and $A$ in the usual channels with SM fermions in the final state are significantly affected in parameter regions where decays of $H$ and $A$ into supersymmetric particles are possible. In particular, we have discussed decays into charginos and neutralinos as well as decays into staus. Furthermore, decays of the heavy $\mathcal{C} \mathcal{P}$-even Higgs boson into a pair of light $\mathcal{C} \mathcal{P}$-even Higgs bosons can be important. We encourage ATLAS and CMS to enhance the sensitivity of their searches for MSSM Higgs bosons by performing also dedicated searches for Higgs decays into SUSY particles and into a pair of lighter Higgs bosons.

We have also defined the light stop scenario, which has $m_{\tilde{t}_{1}} \approx 325 \mathrm{GeV}$ and $m_{\tilde{t}_{2}} \approx 670 \mathrm{GeV}$. The stops give a sizable contribution to the $\sigma(g g \rightarrow h)$ production rate. Similarly, we define the light stau scenario, where the light staus can enhance $\Gamma(h \rightarrow \gamma \gamma)$ substantially at high values of $\tan \beta$. We have furthermore proposed the $\tau$-phobic Higgs scenario, which exhibits potentially sizable variations of $\Gamma(h \rightarrow b \bar{b})$ and $\Gamma(h \rightarrow \tau \tau)$ with respect to their SM values. For the $m_{h}^{\max }, m_{h}^{\bmod }$ and light stop scenarios we propose to investigate several values (and in particular both signs) of the parameter $\mu$, which has an important impact on the bottom Yukawa coupling via the corrections involving the quantity $\Delta_{b}$.

Finally, we define the low- $M_{H}$ scenario, which interprets the heavy $\mathcal{C P}$-even Higgs boson as the newly discovered state at $\sim 125.5 \mathrm{GeV}$. Since this scenario by definition requires a low value of $M_{A}$, we keep $M_{A}$ fixed and instead vary $\mu$ as a free parameter, i.e. the $\mu-\tan \beta$ parameter space is investigated. In most of the allowed parameter space the mass of the heavy $\mathcal{C} \mathcal{P}$-even Higgs boson is close to $125.5 \mathrm{GeV}$, and its production and decay rates are SM-like. The light $\mathcal{C} \mathcal{P}$-even Higgs boson, on the other hand, has heavily suppressed couplings to gauge bosons and a mass that is typically below the LEP limit for a SM-like Higgs. The low $-M_{H}$ scenario is characterized by a particularly rich phenomenology, since all five MSSM Higgs bosons are light. Besides the searches for neutral MSSM Higgs bosons in $\tau^{+} \tau^{-}$final states also charged Higgs boson searches have a high sensitivity for probing this scenario. This scenario could therefore serve also as a useful benchmark for (light) charged Higgs boson searches in the MSSM.

Acknowledgements We thank C. Acereda Ortiz for discussions on the decay rates of $H \rightarrow h h$ and Y. Linke for discussions on the 
$m_{h}^{\text {mod }}$ and low $-M_{H}$ scenarios. We thank P. Bechtle and T. Stefaniak for discussions on HiggsBounds. This work has been supported by the Collaborative Research Center SFB676 of the DFG, "Particles, Strings, and the Early Universe". The work of S.H. was partially supported by CICYT (grant FPA 2010-22163-C02-01) and by the Spanish MICINN's Consolider-Ingenio 2010 Programme under grant Mul-
tiDark CSD2009-00064. The work of O.S. is supported by the Swedish Research Council (VR) through the Oskar Klein Centre. Fermilab is operated by Fermi Research Alliance, LLC under Contract No. DEAC02-07CH11359 with the U.S. Department of Energy. Work at ANL is supported in part by the U.S. Department of Energy under Contract No. DE-AC02-06CH11357.

\section{Appendix: Summary of parameter values}

Table 1 Summary of parameter values for the proposed benchmark scenarios, given in the on-shell (OS) scheme unless otherwise noted. Numbers in parentheses refer to calculations with $\Delta_{\tau}$ effects included in the stau mass evaluation (see the description of the light stau scenario for details). Dimensionful quantities are given in $\mathrm{GeV}$

\begin{tabular}{|c|c|c|c|c|c|c|c|}
\hline Parameter & $m_{h}^{\max }$ & $m_{h}^{\bmod +}$ & $m_{h}^{\bmod -}$ & light stop & light stau & $\tau$-phobic & low $-M_{H}$ \\
\hline$m_{t}$ & 173.2 & 173.2 & 173.2 & 173.2 & 173.2 & 173.2 & 173.2 \\
\hline$M_{A}$ & varied & varied & varied & varied & varied & varied & 110 \\
\hline $\tan \beta$ & varied & varied & varied & varied & varied & varied & varied \\
\hline$M_{\text {SUSY }}$ & 1000 & 1000 & 1000 & 500 & 1000 & 1500 & 1500 \\
\hline$M_{\tilde{l}_{3}}$ & 1000 & 1000 & 1000 & 1000 & $245(250)$ & 500 & 1000 \\
\hline$X_{t}^{\mathrm{OS}} / M_{\mathrm{SUSY}}$ & 2.0 & 1.5 & -1.9 & 2.0 & 1.6 & 2.45 & 2.45 \\
\hline$X_{t}^{\overline{\mathrm{MS}}} / M_{\mathrm{SUSY}}$ & $\sqrt{6}$ & 1.6 & -2.2 & 2.2 & 1.7 & 2.9 & 2.9 \\
\hline$A_{t}$ & \multicolumn{7}{|c|}{ Given by $A_{t}=X_{t}+\mu \cot \beta$} \\
\hline$A_{b}$ & $=A_{t}$ & $=A_{t}$ & $=A_{t}$ & $=A_{t}$ & $=A_{t}$ & $=A_{t}$ & $=A_{t}$ \\
\hline$A_{\tau}$ & $=A_{t}$ & $=A_{t}$ & $=A_{t}$ & $=A_{t}$ & 0 & 0 & $=A_{t}$ \\
\hline$\mu$ & 200 & 200 & 200 & 350 & $500(450)$ & 2000 & varied \\
\hline$M_{1}$ & \multicolumn{7}{|c|}{ Fixed by GUT relation to $M_{2}$} \\
\hline$M_{2}$ & 200 & 200 & 200 & 350 & $200(400)$ & 200 & 200 \\
\hline$m_{\tilde{g}}$ & 1500 & 1500 & 1500 & 1500 & 1500 & 1500 & 1500 \\
\hline$M_{\tilde{q}_{1,2}}$ & 1500 & 1500 & 1500 & 1500 & 1500 & 1500 & 1500 \\
\hline$M_{\tilde{l}_{1,2}}$ & 500 & 500 & 500 & 500 & 500 & 500 & 500 \\
\hline$A_{f \neq t, b, \tau}$ & 0 & 0 & 0 & 0 & 0 & 0 & 0 \\
\hline
\end{tabular}

\section{References}

1. G. Aad et al. (ATLAS Collaboration), Phys. Lett. B 716, 1 (2012). arXiv:1207.7214 [hep-ex]

2. S. Chatrchyan et al. (CMS Collaboration), Phys. Lett. B 716, 30 (2012). arXiv:1207.7235 [hep-ex]

3. ATLAS Collaboration, ATLAS-CONF-2013-012

4. CMS Collaboration, CMS-PAS-HIG-2013-001

5. S.L. Glashow, Nucl. Phys. B 22, 579 (1961)

6. S. Weinberg, Phys. Rev. Lett. 19, 19 (1967)

7. A. Salam, in Proceedings of the 8th Nobel Symposium, Stockholm, ed. by N. Svartholm (1968)

8. H. Nilles, Phys. Rep. 110, 1 (1984)

9. H. Haber, G. Kane, Phys. Rep. 117, 75 (1985)

10. R. Barbieri, Riv. Nuovo Cimento 11, 1 (1988)

11. A. Djouadi, Phys. Rep. 459, 1 (2008). arXiv:hep-ph/0503173

12. S. Heinemeyer, Int. J. Mod. Phys. A 21, 2659 (2006). arXiv:hep-ph/0407244

13. S. Heinemeyer, W. Hollik, G. Weiglein, Phys. Rep. 425, 265 (2006). arXiv:hep-ph/0412214

14. G. Degrassi, S. Heinemeyer, W. Hollik, P. Slavich, G. Weiglein, Eur. Phys. J. C 28, 133 (2003). arXiv:hep-ph/0212020
15. S. Heinemeyer, O. Stål, G. Weiglein, Phys. Lett. B 710, 201 (2012). arXiv:1112.3026 [hep-ph]

16. M. Carena, S. Gori, N. Shah, C.E.M. Wagner, J. High Energy Phys. 1203, 014 (2012). arXiv:1112.3336 [hep-ph]

17. M. Carena, S. Gori, N. Shah, C.E.M. Wagner, L.-T. Wang, J. High Energy Phys. 1207, 175 (2012). arXiv: 1205.5842 [hep-ph]

18. M. Carena, I. Low, C.E.M. Wagner, J. High Energy Phys. 1208, 060 (2012). arXiv:1206.1082 [hep-ph]

19. M. Carena, S. Gori, I. Low, N. Shah, C.E.M. Wagner, J. High Energy Phys. (to appear). arXiv:1211.6136 [hep-ph]

20. R. Benbrik, M. Gomez Bock, S. Heinemeyer, O. Stål, G. Weiglein, L. Zeune, Eur. Phys. J. C 72, 2171 (2012). arXiv:1207.1096 [hep-ph]

21. P. Bechtle, S. Heinemeyer, O. Stål, T. Stefaniak, G. Weiglein, L. Zeune, arXiv:1211.1955 [hep-ph]

22. L. Hall, D. Pinner, J. Ruderman, J. High Energy Phys. 1204, 131 (2012). arXiv:1112.2703 [hep-ph]

23. H. Baer, V. Barger, A. Mustafayev, Phys. Rev. D 85, 075010 (2012). arXiv:1112.3017 [hep-ph]

24. A. Arbey, M. Battaglia, A. Djouadi, F. Mahmoudi, J. Quevillon, Phys. Lett. B 708, 162 (2012). arXiv:1112.3028 [hep-ph] 
25. P. Draper, P. Meade, M. Reece, D. Shih, Phys. Rev. D 85, 095007 (2012). arXiv:1112.3068 [hep-ph]

26. A. Arbey, M. Battaglia, A. Djouadi, F. Mahmoudi, J. High Energy Phys. 1209, 107 (2012). arXiv:1207.1348 [hep-ph]

27. A. Arbey, M. Battaglia, A. Djouadi, F. Mahmoudi, Phys. Lett. B 720, 153 (2013). arXiv:1211.4004 [hep-ph]

28. M. Cahill-Rowley, J. Hewett, A. Ismail, T. Rizzo, Phys. Rev. D 86, 075015 (2012). arXiv:1206.5800 [hep-ph]

29. S. Akula, P. Nath, G. Peim, Phys. Lett. B 717, 188 (2012). arXiv:1207.1839 [hep-ph]

30. S. Antusch, L. Calibbi, V. Maurer, M. Monaco, M. Spinrath, J. High Energy Phys. 1301, 187 (2013). arXiv:1207.7236 [hep-ph]

31. U. Haisch, F. Mahmoudi, J. High Energy Phys. 1301, 061 (2013). arXiv: 1210.7806 [hep-ph]

32. M. Cabrera, J. Casas, R.R. de Austri, arXiv:1212.4821 [hep-ph]

33. R. Gupta, M. Montull, F. Riva, J. High Energy Phys. 1304, 132 (2013). arXiv:1212.5240 [hep-ph]

34. A. Chakraborty, B. Das, J. Diaz-Cruz, D. Ghosh, S. Moretti, P. Poulose, arXiv:1301.2745 [hep-ph]

35. A. Bottino, N. Fornengo, S. Scopel, Phys. Rev. D 85, 095013 (2012). arXiv:1112.5666 [hep-ph]

36. M. Drees, Phys. Rev. D 86, 115018 (2012). arXiv:1210.6507 [hep-ph]

37. R. Barate et al. (LEP Working Group for Higgs boson searches and ALEPH DELPHI and L3 and OPAL Collaborations), Phys. Lett. B 565, 61 (2003). arXiv:hep-ex/0306033

38. B. Petersen (ATLAS Collaboration), talk given at HCP2012, see: http://kds.kek.jp/materialDisplay.py?contribId=46\&sessionId= 20\&materialId=slides \& $\operatorname{confId}=9237$

39. R. Gray (CMS Collaboration), talk given at HCP2012, see: http://kds.kek.jp/materialDisplay.py?contribId=48\&sessionId= 20\& materialId $=$ slides $\&$ confId $=9237$

40. S. Schael et al., (ALEPH, DELPHI, L3, and OPAL Collaborations, and the LEP Working Group for Higgs Boson Searches), Eur. Phys. J. C 47, 547 (2006). arXiv:hep-ex/0602042

41. M. Carena, S. Heinemeyer, C. Wagner, G. Weiglein, arXiv:hep$\mathrm{ph} / 9912223$

42. M. Carena, S. Heinemeyer, C. Wagner, G. Weiglein, Eur. Phys. J. C 26, 601 (2003). arXiv:hep-ph/0202167

43. S. Heinemeyer, W. Hollik, G. Weiglein, J. High Energy Phys. 0006, 009 (2000). arXiv:hep-ph/9909540

44. M. Carena, S. Heinemeyer, C. Wagner, G. Weiglein, Eur. Phys. J. C 45, 797 (2006). arXiv:hep-ph/0511023

45. M. Carena, P. Chankowski, S. Pokorski, C. Wagner, Phys. Lett. B 441, 205 (1998). arXiv:hep-ph/9805349

46. S. Heinemeyer, W. Hollik, G. Weiglein, Eur. Phys. J. C 9, 343 (1999). arXiv:hep-ph/9812472

47. J. Espinosa, I. Navarro, Nucl. Phys. B 615, 82 (2001). arXiv: hep-ph/0104047

48. M. Carena, H. Haber, S. Heinemeyer, W. Hollik, C. Wagner, G. Weiglein, Nucl. Phys. B 580, 29 (2000). arXiv:hep-ph/0001002

49. S. Heinemeyer, W. Hollik, G. Weiglein, Comput. Phys. Commun. 124, 76 (2000). arXiv:hep-ph/9812320

50. T. Hahn, S. Heinemeyer, W. Hollik, H. Rzehak, G. Weiglein, Comput. Phys. Commun. 180, 1426 (2009); see http://www.feynhiggs.de

51. M. Frank, T. Hahn, S. Heinemeyer, W. Hollik, H. Rzehak, G. Weiglein, J. High Energy Phys. 0702, 047 (2007). arXiv:hep-ph/0611326

52. J. Lee, A. Pilaftsis, M. Carena, S. Choi, M. Drees, J. Ellis, C. Wagner, Comput. Phys. Commun. 156, 283 (2004). arXiv:hep-ph/0307377

53. J. Lee, M. Carena, J. Ellis, A. Pilaftsis, C. Wagner, Comput. Phys. Commun. 180, 312 (2009). arXiv:0712.2360 [hep-ph]

54. J. Lee, M. Carena, J. Ellis, A. Pilaftsis, C. Wagner, arXiv:1208.2212 [hep-ph]
55. J. Casas, J. Espinosa, M. Quirós, A. Riotto, Nucl. Phys. B 3, 466 (1995)

56. J. Casas, J. Espinosa, M. Quirós, A. Riotto, Nucl. Phys. B 439, 466 (1995). arXiv:hep-ph/9407389

57. M. Carena, J. Espinosa, M. Quirós, C. Wagner, Phys. Lett. B 355, 209 (1995). arXiv:hep-ph/9504316

58. M. Carena, M. Quirós, C. Wagner, Nucl. Phys. B 461, 407 (1996). arXiv:hep-ph/9508343

59. S. Martin, Phys. Rev. D 65, 116003 (2002). arXiv:hep-ph/ 0111209

60. S. Martin, Phys. Rev. D 66, 096001 (2002). arXiv:hep-ph/ 0206136

61. S. Martin, Phys. Rev. D 67, 095012 (2003). arXiv:hep-ph/ 0211366

62. S. Martin, Phys. Rev. D 68, 075002 (2003). arXiv:hep-ph/ 0307101

63. S. Martin, Phys. Rev. D 70, 016005 (2004). arXiv:hep-ph/ 0312092

64. S. Martin, Phys. Rev. D 71, 016012 (2005). arXiv:hep-ph/ 0405022

65. S. Martin, Phys. Rev. D 71, 116004 (2005). arXiv:hep-ph/ 0502168

66. S. Martin, D. Robertson, Comput. Phys. Commun. 174, 133 (2006). arXiv:hep-ph/0501132

67. S. Martin, Phys. Rev. D 75, 055005 (2007). arXiv:hep-ph/ 0701051

68. R. Harlander, P. Kant, L. Mihaila, M. Steinhauser, Phys. Rev. Lett. 100, 191602 (2008). Phys. Rev. Lett. 101, 039901 (2008). arXiv:0803.0672 [hep-ph]

69. R. Harlander, P. Kant, L. Mihaila, M. Steinhauser, J. High Energy Phys. 1008, 104 (2010). arXiv:1005.5709 [hep-ph]

70. S. Heinemeyer, W. Hollik, G. Weiglein, Phys. Lett. B 455, 179 (1999). arXiv:hep-ph/9903404

71. B. Allanach, A. Djouadi, J. Kneur, W. Porod, P. Slavich, J. High Energy Phys. 0409, 044 (2004). arXiv:hep-ph/0406166

72. K. Williams, H. Rzehak, G. Weiglein, Eur. Phys. J. C 71, 1669 (2011). arXiv:1103.1335 [hep-ph]

73. R. Hempfling, Phys. Rev. D 49, 6168 (1994)

74. L. Hall, R. Rattazzi, U. Sarid, Phys. Rev. D 50, 7048 (1994). arXiv:hep-ph/9306309

75. M. Carena, M. Olechowski, S. Pokorski, C. Wagner, Nucl. Phys. B 426, 269 (1994). arXiv:hep-ph/9402253

76. M. Carena, D. Garcia, U. Nierste, C. Wagner, Nucl. Phys. B 577, 577 (2000). arXiv:hep-ph/9912516

77. H. Eberl, K. Hidaka, S. Kraml, W. Majerotto, Y. Yamada, Phys. Rev. D 62, 055006 (2000). arXiv:hep-ph/9912463

78. J. Guasch, P. Häfliger, M. Spira, Phys. Rev. D 68, 115001 (2003). arXiv:hep-ph/0305101

79. M. Carena, S. Mrenna, C. Wagner, Phys. Rev. D 60, 075010 (1999). arXiv:hep-ph/9808312

80. M. Carena, S. Mrenna, C. Wagner, Phys. Rev. D 62, 055008 (2000). arXiv:hep-ph/9907422

81. A. Brignole, G. Degrassi, P. Slavich, F. Zwirner, Nucl. Phys. B 643, 79 (2002). arXiv:hep-ph/0206101

82. G. Degrassi, A. Dedes, P. Slavich, Nucl. Phys. B 672, 144 (2003). arXiv:hep-ph/0305127

83. S. Heinemeyer, W. Hollik, H. Rzehak, G. Weiglein, Eur. Phys. J. C 39, 465 (2005). arXiv:hep-ph/0411114

84. S. Heinemeyer, W. Hollik, H. Rzehak, G. Weiglein, arXiv:hep-ph/0506254

85. L. Hofer, U. Nierste, D. Scherer, J. High Energy Phys. 0910, 081 (2009). arXiv:0907.5408 [hep-ph]

86. D. Noth, M. Spira, Phys. Rev. Lett. 101, 181801 (2008). arXiv:0808.0087 [hep-ph]

87. D. Noth, M. Spira, J. High Energy Phys. 1106, 084 (2011). arXiv:1001.1935 [hep-ph] 
88. A. Crivellin, C. Greub, Phys. Rev. D 87, 015013 (2013). arXiv: 1210.7453 [hep-ph]

89. S. Gennai et al., Eur. Phys. J. C 52, 383 (2007). arXiv:0704.0619 [hep-ph]

90. M. Hashemi et al., arXiv:0804.1228 [hep-ph]

91. G. Abbiendi et al. (OPAL Collaboration), Eur. Phys. J. C 35, 1 (2004). arXiv:hep-ex/0401026

92. Tevatron Electroweak Working Group, the CDF and DØ Collaborations, arXiv:1107.5255 [hep-ex]

93. A. Djouadi, J. Kalinowski, M. Spira, Comput. Phys. Commun. 108, 56 (1998). hep-ph/9704448

94. P. Bechtle, O. Brein, S. Heinemeyer, G. Weiglein, K. Williams, Comput. Phys. Commun. 181, 138 (2010). arXiv:0811.4169 [hep-ph]

95. P. Bechtle, O. Brein, S. Heinemeyer, G. Weiglein, K. Williams, Comput. Phys. Commun. 182, 2605 (2011). arXiv:1102.1898 [hep-ph]

96. P. Bechtle, O. Brein, S. Heinemeyer, O. Stål, T. Stefaniak, G. Weiglein, K. Williams, arXiv:1301.2345 [hep-ph]

97. P. Bechtle, O. Brein, S. Heinemeyer, O. Stål, T. Stefaniak, G. Weiglein, K. Williams, Manual for HiggsBounds 4.0.0. http://higgsbounds.hepforge.org

98. CMS Collaboration, CMS-PAS-HIG-12-050

99. G. Aad et al. (ATLAS Collaboration), arXiv:1211.6956 [hep-ex]

100. G. Aad et al. (ATLAS Collaboration), J. High Energy Phys. 1206, 039 (2012). arXiv:1204.2760 [hep-ex]

101. S. Chatrchyan et al. (CMS Collaboration), J. High Energy Phys. 1207, 143 (2012). arXiv:1205.5736 [hep-ex]

102. CMS Collaboration, CMS-PAS-HIG-12-045

103. K. Williams, G. Weiglein, Phys. Lett. B 660, 217 (2008). arXiv:0710.5320 [hep-ph]

104. M. Bisset, J. Li, N. Kersting, F. Moortgat, S. Moretti, J. High Energy Phys. 0908, 037 (2009). arXiv:0709.1029 [hep-ph]

105. M. Bisset, F. Moortgat, S. Moretti, Eur. Phys. J. C 30, 419 (2003). arXiv:hep-ph/0303093

106. F. Moortgat, S. Abdullin, D. Denegri, arXiv:hep-ph/0112046

107. W. Altmannshofer, M. Carena, N. Shah, F. Yu, arXiv:1211.1976 [hep-ph]

108. S. Chatrchyan et al. (CMS Collaboration), arXiv:1302.2892 [hep-ex]

109. M. Carena, S. Gori, A. Juste, A. Menon, C.E.M. Wagner, L.-T. Wang, J. High Energy Phys. 1207, 091 (2012). arXiv:1203.1041 [hep-ph]

110. A. Djouadi, Phys. Lett. B 435, 101 (1998). arXiv:hep-ph/ 9806315

111. J. Ellis, M. Gaillard, D. Nanopoulos, Nucl. Phys. B 106, 292 (1976)
112. M. Shifman, A. Vainshtein, M. Voloshin, V. Zakharov, Sov. J. Nucl. Phys. 30, 711 (1979) [Yad. Fiz. 30, 1368 (1979)]

113. K. Blum, R. D’Agnolo, J. Fan, J. High Energy Phys. 1301, 057 (2013). arXiv:1206.5303 [hep-ph]

114. M. Buckley, D. Hooper, Phys. Rev. D 86, 075008 (2012). arXiv:1207.1445 [hep-ph]

115. J. Espinosa, C. Grojean, V. Sanz, M. Trott, J. High Energy Phys. 1212, 077 (2012). arXiv:1207.7355 [hep-ph]

116. G. Aad et al. (ATLAS Collaboration), Phys. Rev. Lett. 109, 211802 (2012). arXiv:1208.1447 [hep-ex]

117. G. Aad et al. (ATLAS Collaboration), Phys. Rev. Lett. 109, 211803 (2012). arXiv:1208.2590 [hep-ex]

118. G. Aad et al. (ATLAS Collaboration), J. High Energy Phys. 1211, 094 (2012). arXiv:1209.4186 [hep-ex]

119. G. Aad et al. (ATLAS Collaboration), Eur. Phys. J. C 72, 2237 (2012). arXiv:1208.4305 [hep-ex]

120. G. Aad et al., arXiv:1209.2102 [hep-ex]

121. G. Aad et al., ATLAS-CONF-2012-166

122. G. Aad et al., ATLAS-CONF-2012-167

123. G. Aad et al., ATLAS-CONF-2013-001

124. G. Aad et al. CMS Collaboration, PAS-SUS-12-023

125. G. Aad et al., PAS-SUS-12-028

126. G. Aad et al., PAS-SUS-12-029

127. G. Aad et al., PAS-SUS-11-030

128. T. Aaltonen et al. (CDF Collaboration), J. High Energy Phys. 1210, 158 (2012). arXiv:1203.4171 [hep-ex]

129. V. Abazov et al. (DØ Collaboration), Phys. Lett. B 665, 1 (2008). arXiv:0803.2263 [hep-ex]

130. J.-J. Cao, Z.-X. Heng, J. Yang, Y.-M. Zhang, J.-Y. Zhu, J. High Energy Phys. 1203, 086 (2012). arXiv:1202.5821 [hep-ph]

131. K. Hagiwara, J. Lee, J. Nakamura, J. High Energy Phys. 1210, 002 (2012). arXiv:1207.0802 [hep-ph]

132. G. Giudice, P. Paradisi, A. Strumia, J. High Energy Phys. 1210, 186 (2012). arXiv:1207.6393 [hep-ph]

133. M. Ajaib, I. Gogoladze, Q. Shafi, Phys. Rev. D 86, 095028 (2012). arXiv:1207.7068 [hep-ph]

134. A. Dabelstein, Nucl. Phys. B 456, 25 (1995). arXiv:hep-ph/ 9503443

135. S. Heinemeyer, W. Hollik, G. Weiglein, Eur. Phys. J. C 16, 139 (2000). arXiv:hep-ph/0003022

136. J. Casas, A. Lleyda, C. Muñoz, Nucl. Phys. B 471, 3 (1996). arXiv:hep-ph/9507294

137. A. Kusenko, P. Langacker, G. Segre, Phys. Rev. D 54, 5824 (1996). arXiv:hep-ph/9602414

138. J. Hisano, S. Sugiyama, Phys. Lett. B 696, 92 (2011) [Erratumibid., B 719, 472 (2013)]. arXiv:1011.0260 [hep-ph]

139. W. Hollik, Priv. communication 